\title{
Performance Analysis of a Cognitive Radio Contention-Aware Channel Selection Algorithm
}

\author{
Agapi Mesodiakaki, Student Member, IEEE, Ferran Adelantado, Member, IEEE, Luis Alonso, Senior \\ Member, IEEE, Christos Verikoukis, Senior Member, IEEE
}

\begin{abstract}
In cognitive radio (CR) networks, due to the ever increasing traffic demands and the limited spectrum resources, it is very likely for several secondary networks (SNs) to coexist and opportunistically use the same primary user $(\mathrm{PU})$ resources. In such scenarios, the ability to distinguish whether a licensed channel is occupied by a PU or by other SNs can significantly improve the spectrum efficiency of the network, while the contention among the SNs already operating on licensed channels with no PU activity, may further affect its throughput and energy efficiency. Therefore, the proper selection of licensed channels could result in notable performance gains. In this paper, we propose a novel contention-aware channel selection algorithm, where the SN under study: i) detects the licensed channels with no PU activity by exploiting cooperative spectrum sensing, ii) estimates the probability of collision in each one, and iii) selects the less contended to access. We provide a detailed analytical model for the throughput and the energy efficiency of the SN and we validate it by means of simulation. We also show the significant performance gains of our proposal in comparison with other relevant state-of-the-art algorithms.
\end{abstract}

Index Terms-Cognitive Radio, Cooperative Spectrum Sensing, Feature Detection, CSMA/CA, Spectrum Overlay, Opportunistic Spectrum Access, Green Communications.

\section{INTRODUCTION}

$\mathbf{C}$ OGNITIVE radio (CR) has received much attention as a possible solution to the spectrum scarcity problem, since it enables the use of licensed channels by unlicensed users (also called secondary users (SUs)) for as long as they remain unused [1]-[3]. Although efficient sensing techniques, security or suitable MAC protocols have been extensively addressed by the research community [4], the initial CR technology immaturity and the subsequent lack of real CR applications has hitherto resulted in a slight interest in the coexistence among secondary networks (SNs).

The opportunistic spectrum sharing, on which SNs' operation is based, relies upon two main premises: the protection of the primary users' (PUs') transmissions and the maximization of the spectrum usage. The former is achieved by applying effective sensing techniques (cooperative or not) [5]-[7]. Therefore, most proposals on CR networks aim at exploring

Copyright (c) 2013 IEEE. Personal use of this material is permitted. However, permission to use this material for any other purposes must be obtained from the IEEE by sending a request to pubs-permissions@ieee.org.

A. Mesodiakaki and L. Alonso are with the Signal Theory and Communications Department, Technical University of Catalonia, Spain. Email:\{agapi.mesodiakaki, luisg\} @ tsc.upc.edu

F. Adelantado is with Open University of Catalonia, Barcelona, Spain. Email: ferranadelantado@uoc.edu

C. Verikoukis is with Telecommunications Technological Centre of Catalonia, Castelldefels, Spain. E-mail: cveri@cttc.es the radio environment and detect transmission opportunities in licensed channels. The ability to identify such opportunities, and the accuracy with which they are detected, are essential to efficiently exploit them. In this context, proposals on suitable sensing and access mechanisms have been stated [8]-[10].

The maximization of the spectrum usage, though, can only be met by implementing efficient coexistence mechanisms among SNs, particularly in congested environments. The new challenges posed by SNs' coexistence are a consequence of the scarce transmission opportunities in densely populated areas, in conjunction with the potential high number of SNs in these scenarios. Hence, as stated in [11], mechanisms for efficient coexistence of more than a single $\mathrm{SN}$ are indispensable.

The key point of such an efficient coexistence is that the contention of two or more SNs over the same channel is allowed, but it impacts decisively on the achievable throughput and energy efficiency. Therefore, a CR-based MAC protocol should i) detect the licensed channels without PU activity, and ii) prioritize the access to the channels with low SU contention.

To that end, in our previous work [12], we proposed a channel selection algorithm that classifies the licensed channels without PU activity according to the number of SUs already operating on them and then selects the less contended one, assuming that all SUs are in saturated conditions. Preliminary simulation results were presented for the performance evaluation of the algorithm. In this paper, we substantially extend our work in [12] by providing the following contributions:

- We present a novel contention-aware channel selection algorithm that: i) exploits cooperative spectrum sensing to detect the free from PU activity licensed channels, ii) for each one estimates the probability of collision, and iii) selects the less contended (i.e., with the lowest probability of collision) to access. It is worth noting that this metric can be applied to various traffic patterns, thus relaxing the assumption of saturated conditions in [12].

- We provide an analytical model for the throughput and the energy efficiency of the SN under study. We validate our model by means of simulation and we study how the time between two consecutive sensing periods affects the aforementioned metrics.

- Finally, we compare the proposed channel selection algorithm with three relevant state-of-the-art algorithms. Simulation results show that the proposed algorithm significantly outperforms its counterparts both in terms of throughput and energy efficiency.

The rest of the paper is organized as follows: In Sections II, III and IV, the related work, the system model and the proposed 
TABLE I

RELATED WORK TABLE

\begin{tabular}{|c||c|c|c|c|c|c|}
\hline & {$[13],[14]$} & {$[15]$} & {$[16]$} & {$[17]$} & {$[18],[19]$} & This work \\
\hline PU activity detection & DB & LS & LS & LS & $x$ & LS \\
\hline SNs' activity detection & DB & LS & LS & LS & LS & LS \\
\hline High flexibility & $x$ & $\checkmark$ & $\checkmark$ & $\checkmark$ & $\checkmark$ & $\checkmark$ \\
\hline Need for SNs infrastructure deployment & $\checkmark$ & $x$ & $x$ & $x$ & $x$ & $x$ \\
\hline Need for SN-Database signaling & $\checkmark$ & $x$ & $x$ & $x$ & $x$ & $x$ \\
\hline PU recovery procedure & $x$ & $x$ & $\checkmark$ & $\checkmark$ & $x$ & $\checkmark$ \\
\hline Number of required transceivers & 1 & 1 & 2 & 1 & 1 & 1 \\
\hline SNs synchronization need & $x$ & $\checkmark$ & $x$ & $x$ & $x$ & $x$ \\
\hline Dedicated common control channel & $x$ & $x$ & $x$ & $\checkmark$ & $x$ & $x$ \\
\hline Multi-channel design & $x$ & $x$ & $x$ & $\checkmark$ & $\checkmark$ & $\checkmark$ \\
\hline Spectrum availability calculation & $x$ & $x$ & $x$ & $x$ & $\checkmark$ & $\checkmark$ \\
\hline
\end{tabular}

channel selection algorithm are respectively described. In Sections V and VI, the throughput and energy efficiency analysis are presented, respectively. Section VII validates the model accuracy by comparing it with the results obtained by means of simulation and evaluates the performance of the proposed algorithm compared to other relevant state-of-the-art algorithms. Finally, concluding remarks are given in Section VIII.

\section{RELATED WORK}

To the best of the authors' knowledge, most approaches in the literature decouple the opportunistic spectrum sharing problem into two subproblems: the detection of PUs' activity, and the contention of the SNs. Both the PUs' and the SNs' activity detection can be relied on information provided either by geographical databases (DBs) or by local sensing (LS) procedures.

The use of DBs to detect the PU and SU activity presents less flexibility, while it requires the deployment of SNs' infrastructure and signaling between SNs and geographical DBs [13], [14].

On the other hand, by using LS for the joint detection of $\mathrm{PU}$ and SU activity, the coexistence problem is tackled in an holistic manner [15]-[17]. In [15], a set of known SNs accesses the channel in a TDMA fashion. However, the proposed algorithm is designed for a single licensed channel, and the throughput analysis exposes details on the channel access but it does not gain insight in the recovery procedure when PUs resume their activity. Additionally, it requires synchronization between the SNs. In [16], the authors address the coexistence problem between SNs, although the proposal is not designed for a multichannel scenario. Furthermore, SUs require two transceivers to operate (one devoted to data and another to sensing).

Being the closest to our work, [17]-[19] focus on multichannel scenarios. In [17], the authors propose a MAC protocol for opportunistic spectrum access that uses two channel selection methods, a uniform and a spectrum opportunitybased. According to the first, each SU chooses a channel randomly, whereas the latter takes into account the different spectrum availability probabilities in the channels. However, the authors assume that each SU can correctly estimate the spectrum availability probability (i.e., the number of active secondary flows). Moreover, they consider a dedicated common control channel for control information exchange among the SUs. In [18], the authors propose two algorithms to rank the channels according to their interference severity in terms of strength and activity. Equivalently, in [19], a new carrier sense multiple access (CSMA) protocol that uses a distributed channel selection scheme is proposed, according to which, the transmitter selects an appropriate channel for transmission based on its interference power measurements in the channels. Nevertheless, unlike our work, [18], [19] do not tackle the coexistence problem in a holistic manner, by jointly designing the detection of PU activity and SNs coexistence, since the original problem is decoupled into a multichannel access problem without PUs. For the reader's convenience, the differences between our work and the state-of-the-art are summarized in Table I.

\section{SYSTEM MODEL}

We consider $M$ licensed channels that are allocated to PUs and can be opportunistically accessed by SUs, as long as they remain unused. We assume that the PU activity follows an exponential on-off traffic model, with the mean durations of on and off periods denoted by $T_{o n}$ and $T_{o f f}$, respectively. While being idle ${ }^{1}$ the licensed channels are further characterized by their SU contention level, i.e., the probability of collision among the SUs that operate on them. In particular, for the licensed channel $k$, the collision probability among the SUs is denoted by $p_{C k}$.

We also consider a highly congested unlicensed channel (e.g., belonging to the industrial, scientific and medical band), which is operated by users with and without cognitive capabilities $^{2}$. Among the users operating on the unlicensed channel, we will focus on $N$ users with cognitive capabilities that, due to the high contention in the unlicensed channel and given that there is information to be transferred among them, they set up an ad hoc SN to exploit the spectrum opportunities in licensed channels. We will refer to these users as SUs, whereas the number of users that operate on the unlicensed channel but do not belong to the $\mathrm{SN}$ is denoted by $N_{\text {unlic }}$.

The SN intends to exploit exclusively the idle licensed channels. However, there are two situations where the operation

\footnotetext{
${ }^{1}$ Although an idle channel implies the absence of any type of activity, in this article, we will characterize a channel as busy or idle only based on the PU activity. Thus, an idle channel may still be occupied by SNs.

${ }^{2}$ We define the term cognitive capabilities as the set of features that confer on users the ability to tune and sense different channels, and transmit over them if they are detected idle.
} 
on the unlicensed channel is inevitable: i) during the initial set up of the $\mathrm{SN}$, the exchange of control information is carried out on the unlicensed channel, and ii) when all the available licensed channels are/become busy. Although it will be detailed in Section IV, it is worth noting that no dedicated common control channel is used, since the licensed channels are shared for both control and data transmissions, and the unlicensed channel is only used as a common control channel in the two situations stated above.

The SN under study consists of a cluster head ${ }^{3}$ [3]-[6], whose role may be assigned to the SUs in a round robin way. The sequential assignment of the cluster head role among the SUs improves the algorithm performance in two ways: i) it achieves energy consumption fairness among the SUs [20], and ii) it limits the negative effect of a selfish cluster head, since this is restricted to the time it takes up this role. Furthermore, we assume that the SUs of the SN under study are adequately close to each other to be exposed to the same channel activity. However, note that their reported sensing results may differ due to false alarm and mis-detection probability.

All SUs that are considered in our system model are equipped with a half duplex transceiver. Thus, even if they are capable of operating over multiple channels, including the licensed channels, they can either transmit or receive over a single channel at any given time. Obviously, the use of a single transceiver is less energy-consuming and costly compared with the use of multiple transceivers and is already considered in some CR devices and prototypes [21].

The SUs' transmissions both in the unlicensed channel and licensed channels use the CSMA/CA access method [22], while the PUs may use their own access method, when accessing the licensed channels (e.g., SC-FDMA in the uplink and OFDMA in the downlink for LTE access).

Although the set of licensed channels sensed by the users of the $\mathrm{SN}$ is higher than one, all the users operate on the same single channel by employing CSMA/CA (in fact, several channels are used, but in a sequential manner, since they have to be vacated when they become busy). Thereby, two objectives are achieved: i) the connectivity between all SUs of the SN is guaranteed, and ii) collisions are avoided (or at least minimized) due to the use of CSMA/CA.

\section{Algorithm DESCRIPTION}

The SN is assumed to be initially located in a highly congested unlicensed channel (shared with other $N_{\text {unlic }}$ users). There, the cluster head initiates a sensing procedure aiming at finding new spectrum opportunities for the $\mathrm{SN}$ in licensed channels. Upon the sensing procedure completion, the sensing information is exchanged over the unlicensed channel (Section IV-A), and a list containing the licensed channels detected idle is constructed. Then, there are two possible cases:

i) All the licensed channels have been sensed busy: The list is empty; the SN stays in the unlicensed channel and another sensing procedure is initiated.

\footnotetext{
${ }^{3}$ We assume that the clustering of the SUs is done beforehand. Nevertheless, it is worth mentioning that this takes place in the unlicensed channel, where all users are able to communicate with each other without the presence of PUs.
}

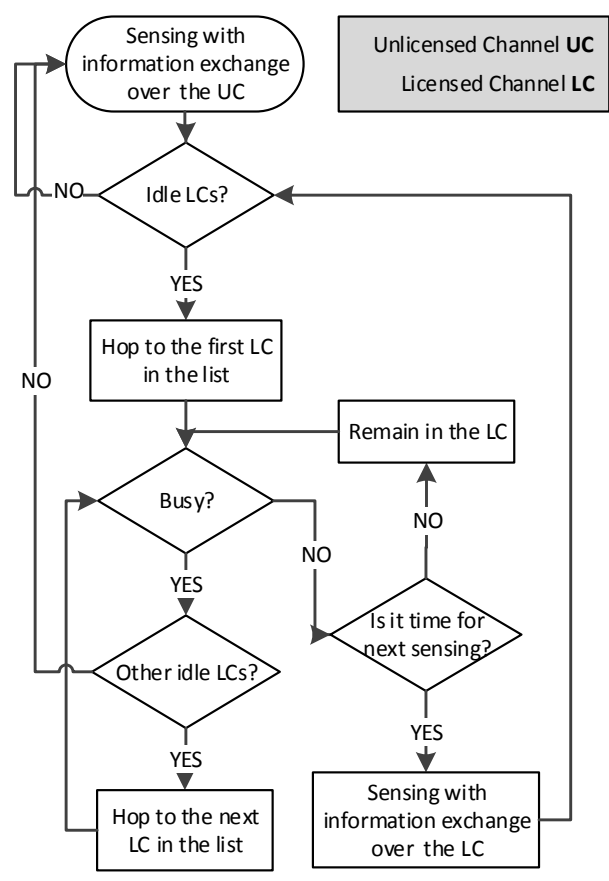

Fig. 1. Protocol flowchart.

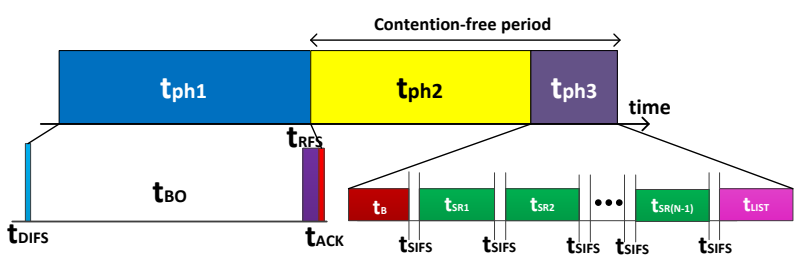

Fig. 2. Time periods of a sensing procedure.

ii) There is at least one licensed channel sensed idle: The list is not empty; the SN hops to the first channel of the list and operates there, as described in Section IV-B.

The protocol flowchart is depicted in Fig. 1 and it is elaborated in the following.

\section{A. Operation on the unlicensed channel}

The operation on the unlicensed channel includes only sensing-related control information exchange. During this procedure the unlicensed channel is used as a common control channel. A sensing procedure can be divided into three periods $\left(t_{p h 1}, t_{p h 2}, t_{p h 3}\right)$, as depicted in Fig. 2.

1) Time period $t_{p h 1}$ : During this period, in order to limit the experienced delay, only the cluster head contends with the other $N_{\text {unlic }}$ users to gain access to the unlicensed channel to carry out the whole process (i.e., the rest $N-1$ users of the SN remain idle). Thus, the cluster head first listens to the channel for a predetermined amount of time $\left(t_{D I F S}\right)$ to determine whether or not another node is transmitting. If no other node transmits, it broadcasts a request for sensing (RFS) packet. Otherwise, it defers its transmission for a random period of time (i.e., back-off time $\left(t_{B O}\right)$ ). The RFS packet defines: i) which licensed channels will be sensed by each SU, ii) the order in which the SUs will report their sensing results to the 
cluster head, and iii) how often the sensing procedure will be triggered. To than end, the following must be noted:

i) All SUs sense the same number of channels in each sensing period, thereby guaranteeing energy consumption fairness. This number is decided during the initial setup, whereas the particular sensing channel assignment is decided and informed by the cluster head with every RFS packet. For this assignment, the cluster head may use information collected in previous periods (while exploiting learning and/or predictive mechanisms) or apply any of the sensing channel assignment algorithms available in the literature [20], [23], [24]. Notice that the use of the round robin algorithm would correspond to the lower performance bound, while any other algorithm, being based on additional information, could improve the SN performance, since the number of channels correctly detected idle would be higher.

ii) Each $\mathrm{SU}$ is assigned a network $i d$ in the setup process (0 to $N-1$ ). Consequently, the cluster head does not need to include in the RFS packet the reporting order of the SUs during $t_{p h 3}$ nor the identity of the next cluster head. In particular, before the beginning of every sensing process, the next cluster head $i d$ is computed as $i d_{\text {next }}=\left(i d_{\text {current }}+1\right) \bmod N$, whereas the reporting order is $\left(i d_{\text {current }}+1\right) \bmod N$ to $\left(i d_{\text {current }}-1\right) \bmod N$. For instance, if $N=4$ and the current cluster head has an $i d$ equal to 2, the next cluster head will have $i d=3$, and the reporting order will be $\{3,0,1\}$. This strategy does not induce any additional overhead.

iii) As the licensed channels state varies along time, the sensing procedure should be repeated periodically to update the channel information. We define $T_{S}$ as the time elapsed between the completion of a sensing procedure and the triggering of the next (by broadcasting a new RFS packet). This value is tightly coupled with the PU activity, namely for fast changing PU activity, a low $T_{S}$ should be chosen to keep the information for every channel updated.

To guarantee the successful transmission of RFS, the algorithm in [25] is applied. Accordingly, one node (i.e., the SU scheduled to send its sensing results first) acts as a leader for the purpose of sending feedback to the cluster head. On erroneous RFS reception, the leader does not send an ACK, prompting a retransmission. On erroneous RFS reception at receivers other than the leader (i.e., at the rest $N-2$ users of the $\mathrm{SN}$ ), the protocol allows negative ACKs from them to collide with the ACK from the leader, thus prompting the cluster head to retransmit the packet.

2) Time period $t_{p h 2}$ : Upon the RFS successful reception, $t_{p h 2}$ begins, with each SU sensing the channels that were assigned to it. In CR networks, as the channels are licensed, it is important to sense a set of channels to have alternatives to hop to in case SUs have to vacate the channel. We should also point out that when a licensed channel is sensed by more than one $\mathrm{SU}$, cooperative spectrum sensing is applied. In our work, the OR fusion rule ${ }^{4}$ is used, which presents low mis-detection and high false alarm probability. In the OR rule, which is the most conservative fusion rule, when at least one of the cooperating SUs senses the licensed channel as busy, the final decision declares a PU is present. Although the application of other fusion rules could achieve better trade-off between false alarm and mis-detection probabilities, the OR rule minimizes the probability of interfering with the PUs, which is the reason why it is selected for our approach.

During sensing $\left(t_{p h 2}\right)$, cyclostationary feature detection is used [4], which enables the SU that senses the licensed channel to distinguish between PUs' and SUs' signals, at the expense of higher complexity and longer sensing time. Since this technique determines the presence of PU signals by extracting their specific features (e.g., pilot signals, cyclic prefixes), it requires prior information about the PU waveforms. However, notice that this is typically known for most standard technologies that operate on licensed channels [26]. Moreover, in coexisting scenarios its use is fundamental, since a simpler technique, unable to distinguish between PUs' and SUs' signals (i.e., energy detection), would result in very low spectrum efficiency, as all the idle channels being used by other SNs, would be considered busy and thus would be avoided.

3) Time period $t_{p h 3}$ : After the sensing has finished, all SUs of the SN hop back to the unlicensed channel to report their sensing results. Given the importance of exchanging them as soon as possible, we consider the reservation of the unlicensed channel for the constant and known period of $t_{p h 2}+t_{p h 3}$, as long as its duration is lower than the maximum tolerable delay $^{5}$.

Hence, the cluster head broadcasts a beacon frame (of duration $t_{B}$ ) asking for the sensing results of the rest of the SUs, as depicted in Fig. 2. Subsequently, each SU waits $t_{S I F S}$ and sends its sensing results $\left(t_{S R}\right)$ to the cluster head in the previously defined order. Thereafter, the cluster head constructs and broadcasts the list $\left(t_{L I S T}\right)$ and the contentionfree period ends.

\section{B. Operation on the licensed channels}

When the SN under study hops to a licensed channel, operates there using CSMA/CA. Thus, all SUs that have a packet to send (belonging to the SN under study or/and to the other coexisting SNs) contend to gain access to the licensed channel. Hence, the operation time of the SN under study on the licensed channel consists of successful transmission, collision and idle slots ${ }^{6}$. This normal CSMA/CA operation on the licensed channel is interrupted in the following cases:

\footnotetext{
${ }^{4}$ In cooperative spectrum sensing, the SUs report their sensing results to a central entity (to the cluster head in our case), which process them and makes a final decision according to a predefined rule, also called fusion rule.

${ }^{5}$ This channel reservation is compatible with existing standards, such as the transmission opportunity (TXOP) in 802.11 [27].

${ }^{6}$ Please note that although we use the term slot, the SUs' access is not slotted since they use CSMA/CA. Yet, we will refer to a slot, as defined in [22], to determine the duration of a successful transmission (successful transmission slot), of a collision (collision slot) or of an idle period (empty slot).
} 


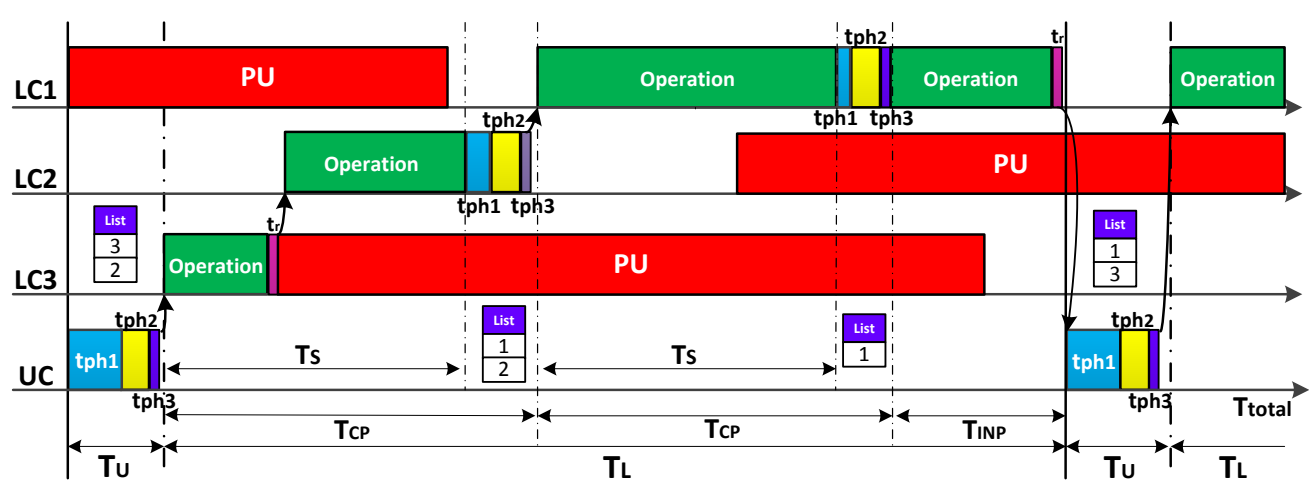

Fig. 3. Example of the SN under study operation.

i) The PU of the licensed channel remains idle and it is time to initiate the next sensing procedure (i.e., $T_{S}$ has elapsed). In this case, only the cluster head contends with the other coexisting SNs to gain access to the licensed channel and trigger a new sensing procedure (consisting again of $t_{p h 1}, t_{p h 2}$ and $t_{p h 3}$, as described in the unlicensed channel operation).

ii) The PU becomes busy earlier than $T_{S}$. In this case, the SUs have to leave the licensed channel immediately in order not to interfere with the PU. The time that the SN requires to detect the PU activity and react accordingly by hopping to the next licensed channel in the list is denoted by $t_{r}$. In case all the channels of the list have been visited and have become busy before $T_{S}$, the SN hops to the unlicensed channel to trigger a new sensing procedure.

\section{Channel selection algorithm}

As cyclostationary feature detection is able to discern between PU and SU activity, after the sensing procedure completion, the cluster head constructs a list containing the licensed channels where no PU activity has been detected (idle channels). These channels may have other SNs operating on them and thus may be characterized by the probability of collision among the SUs.

The main goal of the algorithm is to achieve throughput and energy efficiency improvement by reducing the time spent in highly contended licensed channels. Therefore, the channels in the list are sorted in ascending order by the estimation of their probability of collision among the SUs, $p_{C}$ (i.e., the channel with the lowest $p_{C}$ takes the first place and, thus, higher priority). Notice that as the licensed channels are classified and accessed based on their activity, the SNs (and so the SUs belonging to them) are distributed among the licensed channels, thereby achieving: load balancing over the channels, connectivity for each particular SN, reduction of the coordination signaling burden, and minimization of the need for a dedicated common control channel.

A SU that senses a licensed channel can efficiently estimate $p_{C}$ by simply monitoring the channel activity. Specifically, it is able to understand the collisions and the successful transmissions of the other SNs by listening to their packet exchange [28]. Thus, $p_{C}$ can be measured by counting the number of slots that a successful transmission occurs $\left(C_{\text {succ }}\right)$, as well as the number of slots that a collision of the other
SNs occurs $\left(C_{\text {coll }}\right)$, as in each of these slots a potential packet transmission of the SN under study would have failed [29]. Thus, $p_{C}$ may be expressed as

$$
p_{C}=\frac{C_{\text {succ }}+C_{\text {coll }}}{B}=1-\frac{C_{\text {idle }}}{C_{\text {succ }}+C_{\text {coll }}+C_{\text {idle }}}
$$

where $B$ is the total number of observed slots that also includes the number of idle slots $\left(C_{\text {idle }}\right)$.

The estimation accuracy is highly dependent on the observation time $(B)$ (e.g., for constant SU activity, the longer the observation time, the more accurate the $p_{C}$ ). Therefore, the algorithm should be robust enough to overcome situations of overestimation and/or underestimation of $p_{C}$. To that end, in [12] it is shown that the correct construction of the list is slightly impacted by $p_{C}$ estimation inaccuracy, since it depends more significantly on the comparison between the estimated values and not on the estimated values themselves.

\section{Throughrut AnAlysis}

The throughput of the SN under study may be expressed as

$$
S=\frac{\mathbf{E}[D]}{\mathbf{E}\left[T_{U}\right]+\mathbf{E}\left[T_{L}\right]}
$$

where $\mathbf{E}[D]$ is the expected number of useful bits (i.e., payload) sent by the $\mathrm{SN}$ in a representative time period, $T_{p}$, defined as the sum of the time spent in the unlicensed $\left(T_{U}\right)$ and licensed channels $\left(T_{L}\right)$, until the $\mathrm{SN}$ hops back to the unlicensed. Notice that $T_{p}$ is a random variable, since it depends on the contention in the unlicensed and on the PU activity in the licensed channels (the SN hops back to the unlicensed channel when all the licensed channels previously detected idle become busy). For a better understanding of $T_{p}$, an operation example is given in Fig. 3, where

- $T_{U}$ : Time spent by the $\mathrm{SN}$ in the unlicensed channel, until there is at least one licensed channel sensed idle. Then, the SN hops to the first channel on the list and $T_{L}$ begins.

- $T_{L}$ : Time spent by the $\mathrm{SN}$ in licensed channels, until the moment that there is no licensed channel available and the SN has to hop again to the unlicensed channel for the recovery process. $T_{L}$ may consist of a number of complete periods (e.g., two in Fig. 3) and an incomplete period. During a complete period, the SN operates on licensed channels for $T_{S}$ and a sensing procedure takes place, while in an incomplete period, the $\mathrm{SN}$ operates on 
licensed channels for less than $T_{S}$ and there is no other available licensed channel in the list (i.e., all the licensed channels become busy before $T_{S}$ ). After an incomplete period, the SN hops back to the unlicensed channel, where it will remain for $T_{U}$. Thus, the expected value of $T_{p}$ can be expressed as the sum of the expected values of $T_{U}$ and $T_{L}$.

\section{A. Calculation of $\boldsymbol{E}[D]$}

Prior to further calculations, two key points must be clearly stated. First, the amount of data transmitted over the licensed channels is tightly coupled with the number of available channels and with the time that these channels remain idle. The more the available channels, the more the transmission opportunities for the SN. Second, the SN aims to operate exclusively on licensed channels (after the initial setup on the unlicensed channel) or at least for as long as possible. This mainly depends on $T_{S}$ and the PUs' (in)activity period. If $T_{S}$ is longer compared to the inactivity periods, the probability that all the available channels become busy between two consecutive sensing procedures increases. On the contrary, if $T_{S}$ is considerably shorter, unnecessary sensing procedures are triggered, thereby reducing the SN's effective transmission opportunities.

In this context, the set of ordered channels detected idle after a cooperative sensing procedure of the $\mathrm{SN}$ is denoted by $\mathcal{B}^{7}$. After the sensing procedure, all SUs operate on the first channel in $\mathcal{B}$ for as long as it remains idle. Then, when the channel turns into the busy state, all the users hop to the second channel in $\mathcal{B}$. This operation is repeated until there are no channels available.

Lemma 1. Given a set of channels in $\mathcal{B}$ with activity and inactivity periods independently and exponentially distributed with $T_{\text {on }}$ and $T_{\text {off }}$ mean values respectively, the time elapsed between the beginning and the end (due to PU activity resumption) of the $S N$ operation on the kth channel in $\mathcal{B}$ is denoted by $\tau_{k}$. The probability density function ( $\left.p d f\right)$ of $\tau_{k}$ can be written as in (3) of the next page, where $\mathcal{O}_{k}(t) \in\{$ idle, busy $\}$ is the actual state of the kth channel at time $t$ and $S_{k-1}$ denotes the total time spent in the previous $k-1$ licensed channels,

$$
S_{k-1}=\sum_{j=1}^{k-1} \tau_{j}+(j-1) \delta
$$

where $\delta=t_{r}+t_{s w}$ is the time required to detect the change in licensed channel activity $\left(t_{r}\right)$ and switch to the following channel $\left(t_{s w}\right)$.

\section{Proof: See Appendix A.}

As already mentioned, the $\mathrm{SN}$ exchanges data packets only in the licensed channels. Thus, given the set $\mathcal{B}$ of licensed channels the expected payload sent by the $\mathrm{SN}, \mathbf{E}[D]$, equals to

$$
\mathbf{E}[D]=\sum_{k \in \mathcal{B}} \mathbf{E}\left[N_{\text {pack }_{k}}\right] \mathbf{E}[P]
$$

\footnotetext{
${ }^{7}$ The set $\mathcal{B}$ contains all the licensed channels sensed with no PU activity, ordered by the other SNs' activity; the lower the other SNs' activity in the channel, the lower the channel ordinal in $\mathcal{B}$, and the higher the probability of being visited by the $\mathrm{SN}$.
}

where $\mathbf{E}\left[N_{\text {pack }_{k}}\right]$ denotes the expected number of successfully transmitted packets (or equivalently of successful transmission slots, since a successful transmission slot corresponds to the successful transmission of one packet) by the SN under study in the $k$ th licensed channel during $T_{p}=T_{U}+T_{L}$ and $\mathbf{E}[P]$ the average packet payload size. Then, $\mathbf{E}\left[N_{\text {pack }}\right]$ may be expressed as

$$
\mathbf{E}\left[N_{\text {pack }_{k}}\right]=\frac{\mathbf{E}\left[T_{k}\right]}{\mathbf{E}\left[T_{\text {slot }_{k}}\right]} P_{s_{k}}
$$

where $\mathbf{E}\left[T_{k}\right]$ is the expected operation time (i.e., successful transmission, collision and idle slots) on the $k$ th channel, $P_{s_{k}}{ }^{8}$ the probability of having a successful transmission by the $\mathrm{SN}$ in the $k$ th channel and $\mathbf{E}\left[T_{\text {slot }_{k}}\right]$ denotes the average slot duration in the $k$ th channel.

As previously expounded, a period can be defined as the time between the completion of two consecutive sensing procedures. Henceforth, the periods during which at least one licensed channel remains idle are called complete periods, whereas the periods during which all idle channels change their state are denoted as incomplete periods.

Lemma 2. Given a set of channels $\mathcal{B}$, ordered according to the sensed contention level, the expected operation time on the kth channel $\left(T_{k}\right)$, is given by

$$
\boldsymbol{E}\left[T_{k}\right]=\sum_{x=0}^{\infty} P(X=x)\left((x-1) \boldsymbol{E}\left[T_{k}\right]_{C P}+\boldsymbol{E}\left[T_{k}\right]_{I N P}\right)
$$

where $X$ is the number of successive periods (i.e., $X-1$ complete and one incomplete periods) operating exclusively on licensed channels, and $\boldsymbol{E}\left[T_{k}\right]_{C P}$ and $\boldsymbol{E}\left[T_{k}\right]_{I N P}$ denote the expected operation time of the $S N$ on the kth channel in a complete and an incomplete period, respectively.

Proof: See Appendix B.

Regarding the average slot duration in the $k$ th channel, $\mathbf{E}\left[T_{\text {slot }_{k}}\right]$, it can be easily derived as

$$
\mathbf{E}\left[T_{\text {slot }_{k}}\right]=P_{i_{k}} \sigma+\left(P_{s_{k}}+P_{s_{k}}^{\prime}\right) T_{r s}+\left(P_{c_{k}}+P_{c_{k}}^{\prime}\right) T_{c}
$$

where $P_{i_{k}}$ is the probability of having an idle slot in the $k$ th channel, $P_{c_{k}}$ the one of a collision slot of the SN under study, and $P_{s_{k}}^{\prime}$ and $P_{c_{k}}^{\prime}$ the probability of a successful transmission and collision slot, respectively, of the other SNs in the $k$ th channel. The parameter $\sigma$ denotes the empty slot duration, while $T_{r s}$ and $T_{c}$ the durations of a successful transmission and collision slot. Further details on the calculation of these parameters are included in Appendix C.

\section{B. Calculation of $\boldsymbol{E}\left[T_{U}\right]$}

The time spent in the unlicensed channels is devoted to sense the licensed channels and share the information on their availability. The SUs will not be able to operate on licensed channels if no available channels have been detected.

\footnotetext{
${ }^{8}$ Please note that the proposed model can be applied to any traffic pattern of contending users in the unlicensed channel and licensed channels. Closed form expressions for the traffic-dependent parameters (e.g., probability of a successful transmission, collision and idle slot) can be found in [22], [30] for saturated and non-saturated conditions, respectively. For the reader's convenience, the ones for saturated conditions are derived in Appendix C.
} 


$$
f_{\tau_{k}}(\tau)=\left\{\begin{array}{l}
P\left(\mathcal{O}_{k}(0)=i d l e\right)\left(1-e^{-\frac{S_{k-1}}{T_{o n}}}-e^{-\frac{S_{k-1}}{T_{o f f}}}\right)+e^{-\frac{S_{k-1}}{T_{o n}}} \\
\frac{1}{T_{o f f}} e^{-\frac{\tau}{T_{o f f}}}\left(\left(e^{-\frac{S_{k-1}}{T_{o f f}}}+e^{-\frac{S_{k-1}}{T_{o n}}}-1\right) P\left(\mathcal{O}_{k}(0)=i d l e\right)+\left(1-e^{-\frac{S_{k-1}}{T_{o n}}}\right)\right) \quad \text { if } \tau=0
\end{array}\right.
$$

Hence, the procedure consisting of gaining access to the unlicensed channel, sensing licensed channels and exchanging the information, will be repeated until there is at least one licensed channel detected as idle.

Lemma 3. The expected time spent in the unlicensed channel is given by

$$
\boldsymbol{E}\left[T_{U}\right]=\boldsymbol{E}\left[T_{U_{u n}}\right]\left(\frac{1}{P_{U_{s}}}-1\right)+\boldsymbol{E}\left[T_{U_{s}}\right]
$$

where $P_{U_{s}}=1-\prod_{n=1}^{M}\left(1-P_{s_{i_{d l e}}}\right)$ is the probability that there is at least one licensed channel sensed as idle ( $M$ is the total number of licensed channels that are sensed, with $|\mathcal{B}| \leq M)$, and $T_{U_{u n}}$ and $T_{U_{s}}$ are the time spent when there is not any channel sensed as idle and when there is at least one channel sensed as idle, respectively. The expected values of $T_{U_{u n}}$ and $T_{U_{s}}$ are given by (10) of the next page and

$$
\boldsymbol{E}\left[T_{U_{s}}\right]=\boldsymbol{E}\left[T_{U_{u n}}\right]+t_{s w}
$$

where $M_{u s}$ is the number of licensed channels to be sensed by each $S U$; $t_{s n}$ the time to sense a licensed channel; $t_{s w}$ the time required to switch between two channels; $\sigma, T_{r s}$ and $T_{c}$ the durations of an idle, a successful transmission and a collision slot; $t_{R F S}, t_{A C K}, t_{B}, t_{S R}$ and $t_{L I S T}$ time required to transmit an RFS, ACK, beacon, report, and list packet, respectively; $P_{i}$ the probability of having an idle slot in the unlicensed channel; $P_{c}$ the probability of a collision slot of the SN under study in the unlicensed channel; and $P_{s}^{\prime}$ and $P_{c}^{\prime}$ are the probabilities of a successful transmission and collision slot of the other SNs in the unlicensed channel, respectively.

\section{Proof: See Appendix D.}

\section{Calculation of $\boldsymbol{E}\left[T_{L}\right]$}

The time spent in licensed channels can be divided into two parts: the effective time devoted to data transmission, and the time devoted to sensing, to detect the resumption of the PUs activity and to switch to an alternative channel. Therefore,

$$
\mathbf{E}\left[T_{L}\right]=\mathbf{E}\left[T_{r s n}\right]+\sum_{k \in \mathcal{B}} \mathbf{E}\left[T_{k}\right]
$$

where $\mathbf{E}\left[T_{r s n}\right]$ is the expected time spent in both the reaction periods and the sensing procedures during $T_{p}$ and $\mathbf{E}\left[T_{k}\right]$ is the operation time on the $k$ th channel calculated in (28). For the sake of clarity, the calculation of $\mathbf{E}\left[T_{r s n}\right]$ is detailed in Appendix E.

\section{ENERGY EFFICIENCY ANALYSIS}

The energy efficiency of the SN under study can be expressed as

$$
Q=\frac{\mathbf{E}[D]}{\mathbf{E}\left[E_{U}\right]+\mathbf{E}\left[E_{L}\right]}
$$

where $\mathbf{E}[D]$ has been derived in (5) and $\mathbf{E}\left[E_{U}\right]$ and $\mathbf{E}\left[E_{L}\right]$ are the expected energy consumptions in the unlicensed channel and licensed channels, respectively, during $T_{p}$.

Lemma 4. The expected energy consumed in the unlicensed channel is given by

$$
\boldsymbol{E}\left[E_{U}\right]=\boldsymbol{E}\left[E_{U_{u n}}\right]\left(\frac{1}{P_{U_{s}}}-1\right)+\boldsymbol{E}\left[E_{U_{s}}\right]
$$

where $E_{U_{u n}}$ and $E_{U_{s}}$ are the energy consumptions when there is not any channel sensed as idle and when there is at least one channel sensed as idle, respectively. The expected values of $E_{U_{u n}}$ and $E_{U_{s}}$ are given by (15) of the next page and

$$
\boldsymbol{E}\left[E_{U_{s}}\right]=\boldsymbol{E}\left[E_{U_{u n}}\right]+N \boldsymbol{P}_{s w} t_{s w}
$$

where $\boldsymbol{P}_{\text {trsm }}, \boldsymbol{P}_{\text {rec }}$ and $\boldsymbol{P}_{\text {idle }}$ are the transmission, reception and idle power, while $\boldsymbol{P}_{s n}$ and $\boldsymbol{P}_{s w}{ }^{9}$ denote the sensing power and the power to switch to another channel, respectively.

Proof: See Appendix F.

Then, the expected energy consumed in the licensed channels can be expressed as

$$
\mathbf{E}\left[E_{L}\right]=\mathbf{E}\left[E_{r s n}\right]+\sum_{k \in \mathcal{B}} \mathbf{E}\left[E_{\text {cont }_{k}}\right]
$$

where $\mathbf{E}\left[E_{r s n}\right]$ is the expected energy consumed by the $\mathrm{SN}$ in both the reaction periods and the sensing procedures during $T_{p}$ and $\mathbf{E}\left[E_{\text {cont }_{k}}\right]$ during the contention time in the $k$ th channel. For the sake of clarity, the calculations of $\mathbf{E}\left[E_{r s n}\right]$ and $\mathbf{E}\left[E_{\text {cont }_{k}}\right]$ are detailed in Appendix G.

\section{ANALYSis AND Simulation Results}

\section{A. Simulation scenarios}

In the extensive simulations we executed in MATLAB ${ }^{\mathrm{TM}}$, we consider a SN of $N$ SUs and a set of $M=6$ licensed channels, while $N_{\text {unlic }}=50$. In order to focus on the performance assessment of the channel selection algorithms, we assume ideal channel conditions (i.e., no fading), while all users have the same probability of false alarm and misdetection for all channels equal to 0.01 and 0.1 , respectively. Without loss of generality, we assume that all users both in the unlicensed channel and licensed channels are in saturated conditions (i.e., always having a packet to transmit). Hence, due to the same traffic conditions, only the number of SUs in each licensed channel is sufficient to define its contention level. Thus, we define $N_{S U_{l i c}}$ as the maximum number of SUs of other SNs that operate on a licensed channel (this also corresponds to the maximum probability of collision). The MAC parameters have been selected according to IEEE 802.11g Standard [27], while all simulation parameters are summarized in Table II.

\footnotetext{
${ }^{9}$ The rest of the power consumptions (e.g., by the cluster head to construct the list) can be considered negligible.
} 


$$
\begin{aligned}
& \mathbf{E}\left[T_{U_{u n}}\right]=\frac{P_{i} \sigma+P_{s}^{\prime} T_{r s}+\left(P_{c}+P_{c}^{\prime}\right) T_{c}}{P_{s}}+M_{u s}\left(t_{s r}+t_{s w}\right)+t_{s w}+t_{R F S}+ \\
& +t_{S I F S}(N+1)+t_{A C K}+2 t_{D I F S}+t_{B}+t_{S R}(N-1)+t_{L I S T} \\
& \mathbf{E}\left[E_{U_{u n}}\right]=\frac{N \boldsymbol{P}_{i d l e}\left(P_{i} \sigma+P_{s}^{\prime} T_{r s}+P_{c}^{\prime} T_{c}\right)+P_{c}\left(t_{d a t a}\left(\boldsymbol{P}_{t r s m}+(N-1) \boldsymbol{P}_{r e c}\right)+N \boldsymbol{P}_{i d l e} t_{D I F S}\right)}{P_{s}}+ \\
& +N\left(M_{u s}\left(\boldsymbol{P}_{s n} t_{s n}+\boldsymbol{P}_{s w} t_{s w}\right)+\boldsymbol{P}_{s w} t_{s w}\right)\left(\boldsymbol{P}_{t r s m}+(N-1) \boldsymbol{P}_{r e c}\right)\left(t_{R F S}+t_{B}+t_{L I S T}\right)+ \\
& +\left(\boldsymbol{P}_{\text {trsm }}+\boldsymbol{P}_{\text {rec }}+(N-2) \boldsymbol{P}_{\text {idle }}\right)\left(t_{A C K}+(N-1) t_{S R}\right)+N \boldsymbol{P}_{\text {idle }}\left((N+1) t_{S I F S}+2 t_{D I F S}\right)
\end{aligned}
$$

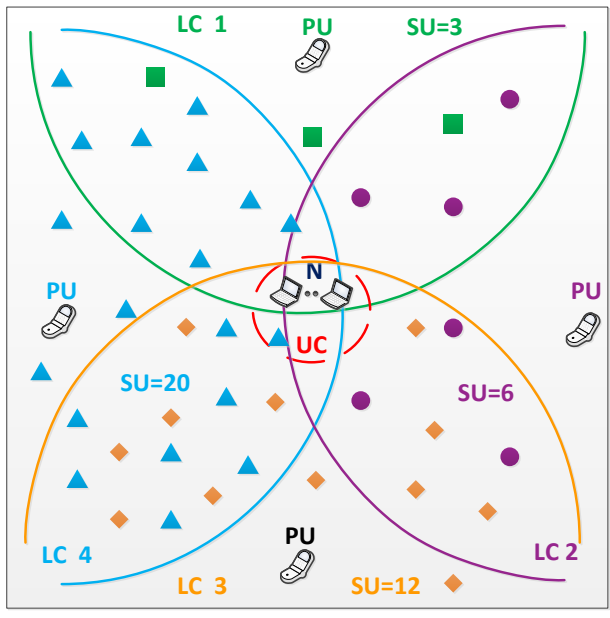

(a)

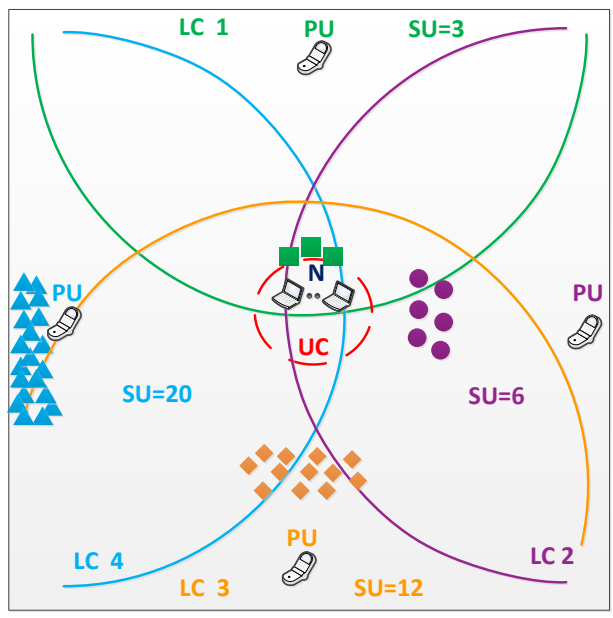

(b)

Fig. 4. (a) Scenario 1, where the SUs of each licensed channel are uniformly distributed around the SN under study and (b) Scenario 2, where the more the SUs on a licensed channel, the further they are located from the SN under study.

TABLE II

SiMULATION VALUES

\begin{tabular}{|l|l||l|l|}
\hline Parameter & Value & Parameter & Value \\
\hline \hline$P_{f a_{k j}} / P_{m d_{k j}}$ & $0.01 / 0.1$ & $m / C W_{m i n}$ & $6 / 16$ \\
$T_{o n}, T_{o f f}$ & $1 \mathrm{~s}$ & $t_{r}, t_{s n}$ & $1 \mathrm{~ms}$ \\
$T X O P$ & $6.016 \mathrm{~ms}$ & $t_{s w}$ & $9 \mathrm{~ms}$ \\
$\sigma$ & $9 \mu \mathrm{s}$ & $t_{S I F S} / t_{D I F S}$ & $10 / 28 \mu \mathrm{s}$ \\
PLCP \& PHY header & $20 \mu \mathrm{s}$ & MAC Header & 34 bytes \\
$l_{A C K}, l_{B}$ & 14 bytes & Payload & 1000 bytes \\
$l_{R F S}$ & 20 bytes & $l_{S R}, l_{L I S T}$ & 16 bytes \\
Ctrl. Trans. Rate & $6 \mathrm{Mbps}$ & Data Trans. Rate & 24 Mbps \\
$\boldsymbol{P}_{r e c}, \boldsymbol{P}_{i d l e}, \boldsymbol{P}_{s n}$ & $1340 \mathrm{~mW}$ & $\boldsymbol{P}_{t r s m}, \boldsymbol{P}_{s w}$ & $1900 \mathrm{~mW}$ \\
\hline
\end{tabular}

Regarding the system topology, the SUs of the SN under study are assumed to be located in the center of a $100 \mathrm{~m}$ $\times 100 \mathrm{~m}$ square region, while the following scenarios are considered:

- Scenario 1: In this general scenario, the SUs of each licensed channel are uniformly distributed around the SN. An example of four licensed channels is given in Fig. 4(a).

- Scenario 2: In this scenario, the more the SUs on a licensed channel, the further they are located from the SN under study (i.e., the SUs of high contended channels are located further, while the SUs of low contended channels are located closer to the SN under study). An example is depicted in Fig. 4(b), where the SUs of licensed channel 1 (LC 1), which is the least contended, are located the closest to the SN, while the users of LC 4, which is the most contended, are located the furthest compared to the SUs operating on LC 2 and LC 3. The purpose of this scenario is to show the dependency of the applied channel selection algorithm on the SUs' topology in each licensed channel. Still, notice that such a scenario could correspond to a heterogeneous network scenario with hotspot traffic at these locations (e.g., in shopping malls).

\section{B. State-of-the-art Algorithms}

Being the closest to our work, the following three channel selection algorithms, previously discussed in Section I, will be adapted to our scenario, to fairly compare them with our approach.

1) Feature detection (FD) algorithm: This algorithm refers to the case where only cyclostationary feature detection is used without any extra estimation technique for the number of contending SUs. In this case, the algorithm is able of distinguishing between channels with PU activity that are avoided and thus are not included in the list, channels with SU activity and channels with no activity at all. For a fair comparison the channels with no activity (neither PU nor SU) will be preferable and, thus, will take the first place in the list. Then, the rest of the channels will be positioned in a random order as in [17], [19].

2) Interference-aware (IA) algorithm: This algorithm proposes the classification of the licensed channels according 


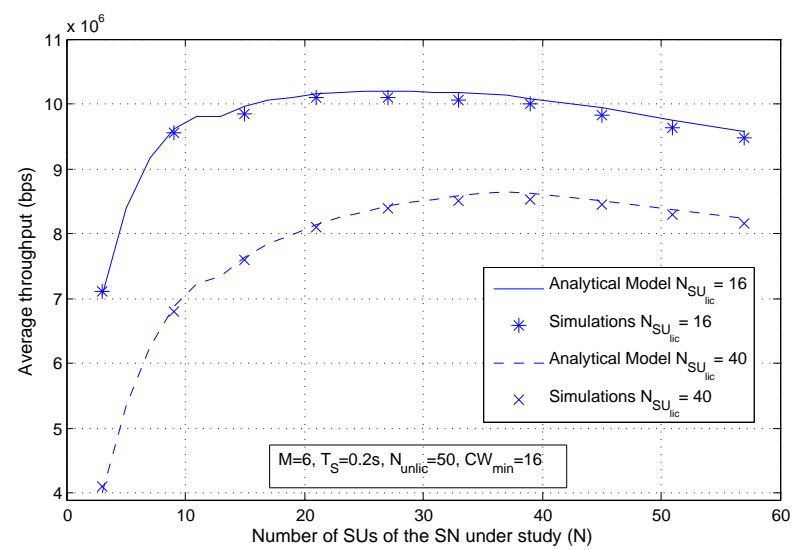

Fig. 5. Throughput versus $N$ for different maximum numbers of competing SUs in any licensed channel, $N_{S U_{l i c}}$.

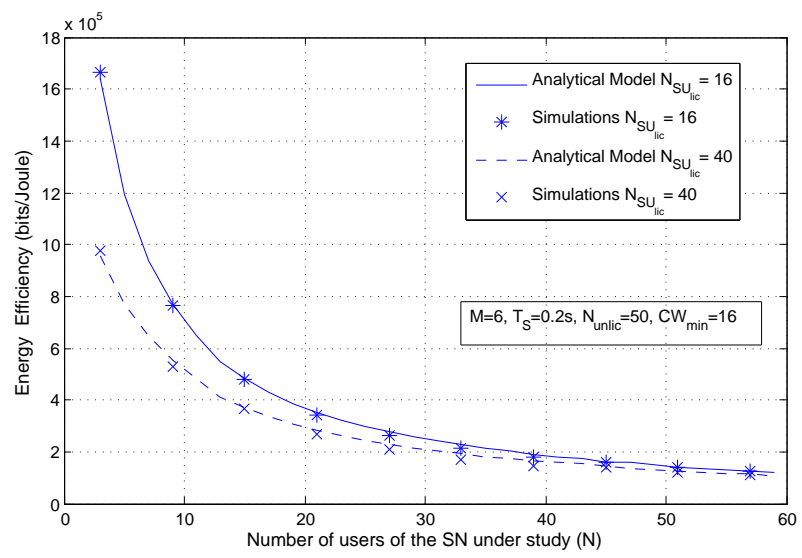

Fig. 6. Energy efficiency versus $N$ for different maximum numbers of competing SUs in any licensed channel, $N_{S U_{l i c}}$.

to their interference level and the selection of the channel with the least interference [18], [19]. In our simulations, the interference is measured by $I=\sum_{s=1}^{N_{k}} G_{s} P_{t r s m}+\sigma_{k}^{2}$, where $N_{k}$ is the number of SUs on the licensed channel $k, \sigma_{k}^{2}$ the variance of the additive white Gaussian noise and $G_{s}$ the channel gain between the SN under study and the user $s$. Then, $G_{s}=\frac{l_{s}}{d_{s}}$, where $l_{s}$ is a random variable representing the lognormal shadowing, $d_{s}$ is the distance between the SN under study and the user $s$ and $n$ denotes the path loss exponent. In our simulations, we consider that $l_{s}$ has mean 0 and variance $8(\mathrm{~dB}), \sigma_{c}^{2}=1 m W$ and $n=3$.

3) Energy detection (ED) Algorithm: Although a comparison between feature detection and energy detection is out of the scope of this paper but can be found in [26], [31], it could provide us with interesting insights that justify the motivation of our work. As previously mentioned, ED, unlike $\mathrm{FD}$, is unable to distinguish if a licensed channel is occupied by a PU or by a SN. For a realistic and fair comparison, in our simulations, we will use the parameter values of [26].

\section{Model validation}

In Fig. 5 and 6, the throughput and the energy efficiency of the $\mathrm{SN}$ versus its number of SUs $(N)$ are depicted analytically and verified by simulations for $N_{S U_{l i c}}=16$ and $N_{S U_{l i c}}=40$. As it can be noticed, as $N$ increases, the throughput is also increased until an upper bound is reached, due to the saturation

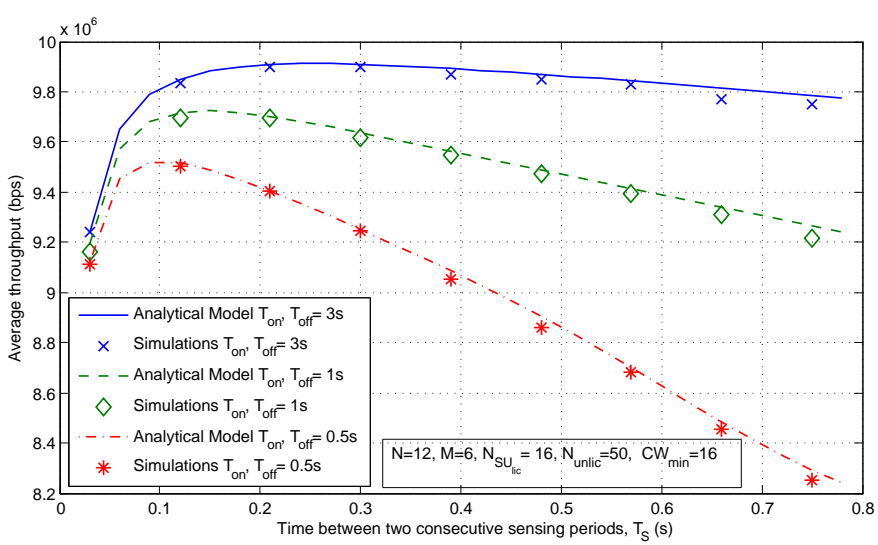

Fig. 7. Throughput versus time between two consecutive sensing periods, $T_{S}$.

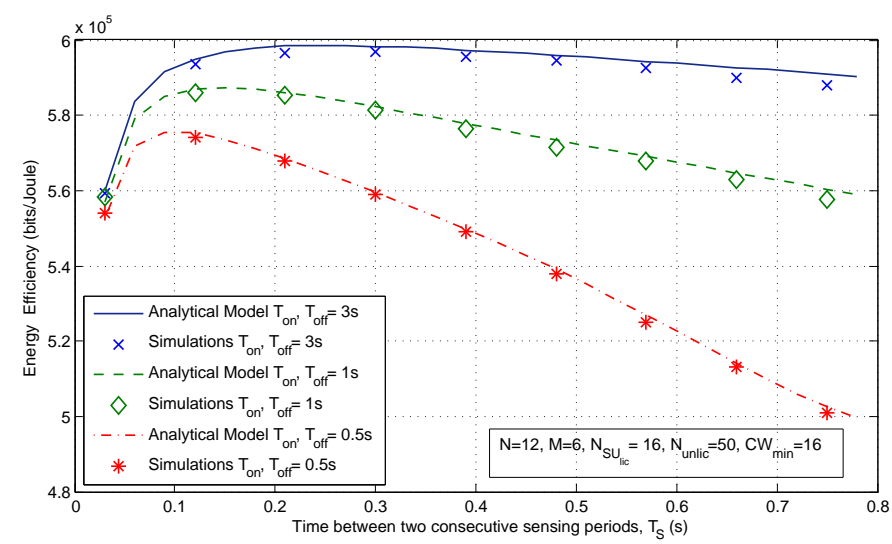

Fig. 8. Energy efficiency versus time between two consecutive sensing periods, $T_{S}$.

of the licensed channel (i.e., saturation throughput) and then it decreases. Notice that this decrease is smooth, due to the trade-off between the detected transmission opportunities and the collision probability. In particular, as $N$ increases, the detection accuracy of the idle channels increases, but so does the collision probability. On the other hand, the energy efficiency of the SN is decreased with the increase of its SUs' number, as the energy consumption increases in a greater extent than the successful bits transmitted by the SN. Furthermore, the less the contention in the licensed channels (i.e., the lower the $N_{S U_{l i c}}$ ), the higher the throughput and the energy efficiency of the SN under study, as it experiences less collisions.

In Fig. 7 and 8 , we study how the time between two consecutive sensing periods $\left(T_{S}\right)$ affects the throughput and the energy efficiency of the $\mathrm{SN}$ for three different values of $T_{o n}$ and $T_{o f f}$. The analytical results are also presented both for throughput and energy efficiency and they are in a good agreement with the simulations. As it can be noticed, there is a maximum throughput and energy efficiency value achieved for each one of the curves. This maximum corresponds to the optimal value of $T_{S}$. For lower values than the optimal, there is a lot of time and energy spent in unnecessary frequent sensing procedures and, thus, less time available for data transmission, whereas for higher ones the list is not updated frequently and, thus, the SN has to switch licensed channels to avoid interfering with the PU. Moreover, as it can be observed, this 


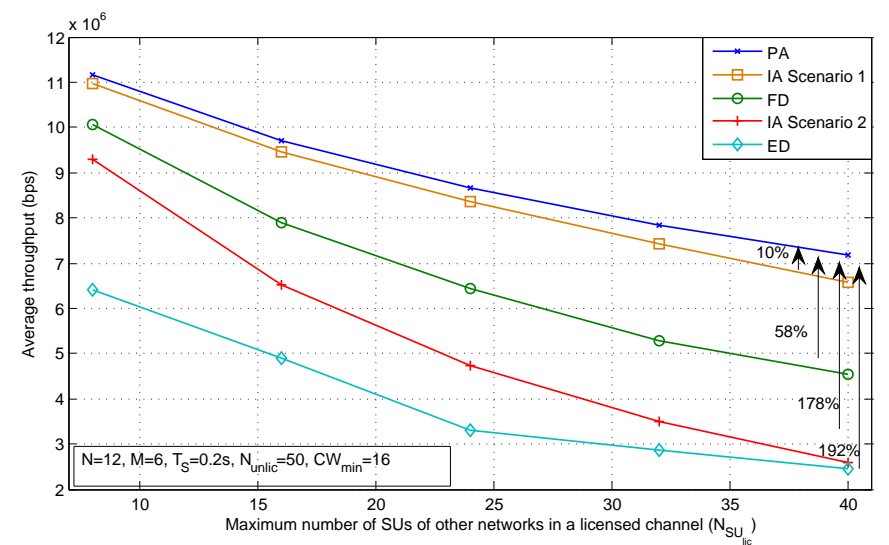

Fig. 9. Throughput versus maximum number of competing SUs in any licensed channel, $N_{S U_{l i c}}$.

optimal value depends on the PU activity pattern. Hence, for slowly-changing PU activity (i.e., high values of $T_{o n}, T_{o f f}$ ), there is no need of frequent sensing procedures and, thus, the optimal value of $T_{S}$ increases, to appropriately adapt to the PU activity. In addition, notice that for a fix value of $T_{S}$, slowly-changing PU activity (i.e., high values of $T_{o n}, T_{o f f}$ ) results in higher throughput and energy efficiency, since the SN operates for a longer amount of time on the channel without PU transmission resumption. On the contrary, for quicklychanging PU activity, the SN has to switch among licensed channels frequently in order not to interfere with the PU, resulting in less time devoted to data transmission and more energy consumption.

\section{Performance evaluation}

In Fig. 9 and 10, the comparison of the proposed algorithm (PA) with the aforementioned state-of-the-art algorithms is given. In particular, the throughput and energy efficiency of the SN under study of all algorithms are respectively depicted with $C W_{\min }=16$, versus the maximum number of SUs in a licensed channel $\left(N_{S U_{l i c}}\right)$. Although it will be detailed later, notice that the IA algorithm performance, unlike the rest of the algorithms, was found to be dependent on the system topology. In addition, it can be observed that, as the parameter $N_{S U_{l i c}}$ increases, both the throughput and energy efficiency of the SN decreases for all algorithms, by virtue of the fact that the contention in the licensed channels increases and so does the energy consumption.

In comparison with FD, PA shows better performance in both throughput and energy efficiency. This stems from the fact that in FD, the SN randomly chooses an idle licensed channel for transmission, thus having higher probability to spend more time in highly contended licensed channels compared to PA. Furthermore, as the parameter $N_{S U_{l i c}}$ increases, the relative gain of PA in both throughput and energy efficiency increases due to its contention-awareness and for $N_{S U_{l i c}}=40$ it can present up to $58 \%$ improvement in throughput and $57 \%$ in energy efficiency.

As far as the IA algorithm is concerned, PA can present up to $178 \%$ improvement in throughput and $175 \%$ in energy efficiency in cases such as the Scenario 2. In that case, the channels are classified in the opposite order than in PA, namely

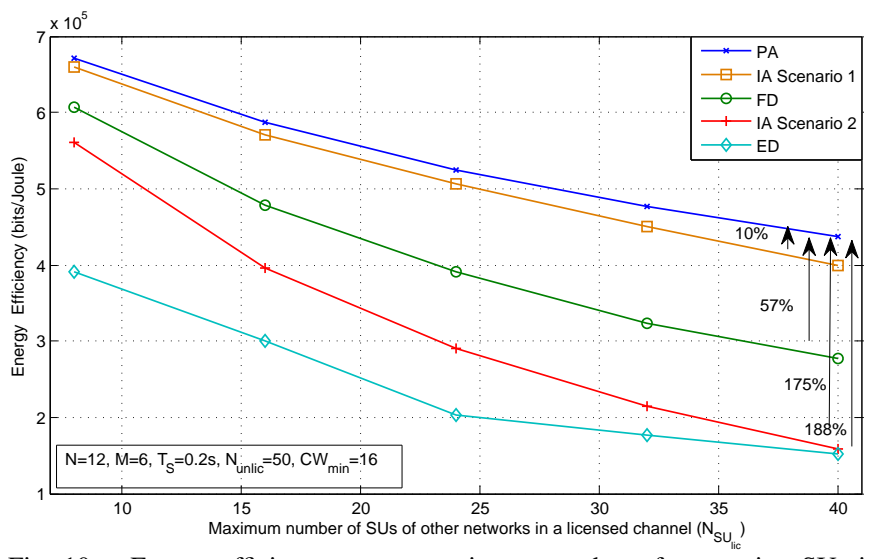

Fig. 10. Energy efficiency versus maximum number of competing SUs in any licensed channel, $N_{S U_{l i c}}$.

the channel with the highest contention will present the least interference and thus, it will be the most preferable by the IA algorithm. Therefore, this scenario defines the maximum gain that can be achieved compared to IA. However, even in cases where the users are uniformly distributed (i.e., in Scenario 1), PA can still present a $10 \%$ improvement, as its performance does not depend on the topology and thus, it achieves higher accuracy in detecting the low contended channels.

Finally, PA significantly outperforms the ED Algorithm, as the spectrum opportunities that are exploited by the ED algorithm are much less than those of PA. The considerably less spectrum efficiency results in a significant degradation of throughput and energy efficiency, as the SN mostly remains in the highly congested unlicensed channel. The gain under high contention in the licensed channels can reach up to $192 \%$ in throughput and $188 \%$ in energy efficiency.

Further experiments were conducted with $C W_{\min }=32$ aiming at studying the impact of the minimum back-off window value on the algorithms' performance (relative graphs are omitted due to lack of space). To that end, it was observed that a higher minimum back-off window value (i.e., with $C W_{\min }=32$ ) results in higher throughput and energy efficiency for all algorithms. This is due to the fact that in this case the SUs have to defer their transmissions for a longer time, when another node transmits and, thus, the collisions are avoided more efficiently. However, this highly depends on the number of contending users; the more the users, the higher the probability of collision and, thus, the higher the minimum back-off window value should be. In addition, it was noticed that PA significantly outperforms the reference algorithms for both the considered values of minimum backoff window, with the highest performance gains being achieved for $C W_{\min }=16$. This stems from the fact that the rest algorithms spend more time in high contended channels, where a very low back off window has a severe impact on the network performance due to the increased number of collisions among the SUs.

\section{CONCLUSION}

In this paper, a novel contention-aware channel selection algorithm has been presented that aims at improving the throughput and energy efficiency of a $\mathrm{SN}$, that coexists with 
other SNs that use the same PU resources. Analytical expressions for the throughput and the energy efficiency of the SN under study have been derived and verified through extensive simulations. Moreover, it has been proved that there is an optimal value for maximum performance for the time between two consecutive sensing periods, which is highly dependent on the PU activity pattern. The PA has been compared to three reference algorithms and it has been shown that it significantly outperforms its counterparts both in terms of throughput and energy efficiency.

\section{APPENDIX A}

PROOF OF LEMMA 1

The (in)activity periods duration in licensed channels are modeled as exponential independent and identically distributed (i.i.d.) random variables. Accordingly, the pdf of the (in)activity period duration of the $k$ th channel can be expressed as $f_{t_{k}}\left(t_{k}\right)=\frac{1}{A} e^{-\frac{t_{k}}{A}}$, where $A$ is equal to the mean value of the inactivity or activity period duration, $T_{o f f}$ and $T_{o n}$, respectively. Hence, if we define $\mathcal{O}_{k}(t) \in\{i d l e, b u s y\}$ as the state of the $k$ th channel at time $t$, the time during which the SN operates on the $k$ th channel $\left(\tau_{k}\right)$ may be written as

$$
\tau_{k}=\left\{\begin{array}{l}
0, \text { if } \mathcal{O}_{k}\left(S_{k-1}\right)=\text { busy } \\
t_{k}-S_{k-1}, \text { otherwise }
\end{array}\right.
$$

where $S_{k-1}$ is the total time spent in the previously visited channels as well as the required time to detect the PUs' activity resumption and the consequent channel switching time, as shown in (4), and $t_{k}$ is the idle state duration given that $\mathcal{O}_{k}\left(S_{k-1}\right)=$ idle. By definition the pdf is equal to the derivative of the cumulative distribution function, i.e., $f_{\tau_{k}}(\tau)=\frac{\partial}{\partial \tau} F_{\tau_{k}}(\tau)$. Taking into account that the channels are divided into actual idle channels detected idle and busy channels erroneously detected idle, the cumulative distribution function is depicted in (19). The first part of the equation models the actual idle channels, whereas the second part models the busy channels. In the case of an idle channel, it will be available for as long as it remains in the idle state. Therefore, the first part can also be expressed as in (20). With regard to the second part of (19), it models a busy channel erroneously detected idle which can be expressed as in (21). In such a case, the channel will only be available for the SN if the state changes before being visited by the $\mathrm{SN}$ and for as long as it remains in the new state (i.e., idle state). In (19) the probability that the $k$ th visited licensed channel is idle at $t=0$ is calculated as

$$
P\left(\mathcal{O}_{k}(0)=i d l e\right)=\frac{P_{i d l e}\left(1-P_{f a_{k}}\right)}{P_{\text {idle }}\left(1-P_{f a_{k}}\right)+P_{m d_{k}}\left(1-P_{\text {idle }}\right)}
$$

where $P_{\text {idle }}$ is the probability of a channel being idle, and $P_{f a_{k}}$ and $P_{m d_{k}}$ the false alarm and mis-detection probability, respectively, resulted from the cooperative sensing on the $k$ th channel. Equivalently, $P\left(\mathcal{O}_{k}(0)=\right.$ busy $)=1-P\left(\mathcal{O}_{k}(0)=\right.$ idle). After some algebra, the pdf of $\tau_{k}$ is given by (3).

\section{APPENDIX B}

\section{PROOF OF LEMMA 2}

We define $X$ as the number of successive periods (i.e., $X-1$ complete periods and one incomplete period) operating exclusively on licensed channels. Thus, the probability of having $x$ successive periods may be expressed as $P(X=x)=$ $P\left(T=T_{C P}\right)^{x-1} P\left(T<T_{C P}\right)$, where $T$ is the time that the SN operates on licensed channels, with a maximum duration of $T_{C P}\left(0 \leq T \leq T_{C P}\right)$ and $P\left(T=T_{C P}\right), P\left(T<T_{C P}\right)$ the probabilities of having a complete and an incomplete period, respectively, with $P\left(T=T_{C P}\right)=1-P\left(T<T_{C P}\right)$ and $P\left(T<T_{C P}\right)$ is given by

$$
P\left(T<T_{C P}\right)=\sum_{n=1}^{M} P\left(n_{i}=n\right) P\left(T<T_{C P} \mid n_{i}=n\right)
$$

where $M$ denotes the total number of licensed channels that are sensed, and $n_{i}$ only the number of channels detected idle (i.e., $n_{i}=|\mathcal{B}|$ ). According to the algorithm description, the random variable $n_{i}$ follows a Poisson binomial distribution and, thus, its probability mass function is given by (24), where $P_{s_{\text {idle } k}}=\left(1-P_{f a_{k}}\right) P_{\text {idle }}+P_{m d_{k}}\left(1-P_{\text {idle }}\right)$ is the probability that the $k$ th licensed channel is sensed idle, with $P_{i d l e}$ the probability of a licensed channel being idle, and $P_{m d_{k}}, P_{f a_{k}}$ the total probabilities of mis-detection and false alarm of the $k$ th channel, respectively. When the OR fusion rule is used to combine the individual sensing reports, $P_{m d_{k}}$ and $P_{f a_{k}}$ are given by (25) and (26), respectively, where $l_{k}$ denotes the number of cooperating SUs that sense the $k$ th channel, $l_{d}$ and $l_{f a}$ are random variables that represent the number of users (with a maximum of $l_{k}$ ) that correctly detect the PU activity in the $k$ th channel or that cause a false alarm, respectively and $P_{m d_{k j}}, P_{f a_{k j}}$ denote the probability of mis-detection and false alarm of user $j$ in the $k$ th channel [6]. Notice that all these parameters depend on the applied sensing channel assignment algorithm, while similar to $n_{i}, l_{d}$ and $l_{f a}$ follow a Poisson binomial distribution. Even though a wide range of hard decision fusion rules for cooperative sensing have been proposed in the literature, here the OR rule is considered. As previously mentioned, the OR rule is the most conservative fusion rule, and consequently its application diminishes the mis-detection probability while increases the false alarm probability. Despite the fact that some other proposals in the literature could achieve better trade-off between false alarm and mis-detection probabilities, the OR rule minimizes the probability of interfering the incumbents of the primary channel. This is the reason why this fusion rule has been chosen. Yet, the analysis presented in this work holds regardless of the applied fusion rule. The selection of an alternative fusion rule would solely result in different expressions in (25) and (26).

Given that there are $n$ licensed channels sensed as idle, an incomplete period takes place when all the $n$ channels become busy before $T_{S}$. In other words, when the SN operates for less than $T_{S}-S_{n-1}$ on the $n$th licensed channel (i.e., the last channel of the list), with $S_{n-1}$ denoting the total time spent in the previous $n-1$ channels. Thus, $P\left(T<T_{C P} \mid n_{i}=n\right)$ is given by (27), where $f_{\tau_{n}}$ is the pdf of $\tau_{n}$ (i.e., operation time on the $n$th visited channel).

The expected operation time on the $k$ th channel, when 


$$
\begin{aligned}
& F_{\tau_{k}}(\tau)=P\left(\tau_{k} \leq \tau\right)=P\left(\tau_{k} \leq \tau \mid \mathcal{O}_{k}(0)=i d l e\right) P\left(\mathcal{O}_{k}(0)=i d l e\right)+P\left(\tau_{k} \leq \tau \mid \mathcal{O}_{k}(0)=\text { busy }\right) P\left(\mathcal{O}_{k}(0)=\text { busy }\right) \\
& P\left(\tau_{k} \leq \tau \mid \mathcal{O}_{k}(0)=i d l e\right)=P\left(\tau_{k} \leq \tau \mid \mathcal{O}_{k}(0)=\text { idle } \cap \mathcal{O}_{k}\left(S_{k-1}\right)=\text { busy }\right) P\left(\mathcal{O}_{k}\left(S_{k-1}\right)=\text { busy } \mid \mathcal{O}_{k}(0)=i d l e\right)+ \\
& +\quad P\left(\tau_{k} \leq \tau \mid \mathcal{O}_{k}(0)=i d l e \cap \mathcal{O}_{k}\left(S_{k-1}\right)=i d l e\right) P\left(\mathcal{O}_{k}\left(S_{k-1}\right)=i d l e \mid \mathcal{O}_{k}(0)=i d l e\right) \\
& P\left(\tau_{k} \leq \tau \mid \mathcal{O}_{k}(0)=\text { busy }\right) \quad=\quad P\left(\tau_{k} \leq \tau \mid \mathcal{O}_{k}(0)=\text { busy } \cap \mathcal{O}_{k}\left(S_{k-1}\right)=\text { busy }\right) P\left(\mathcal{O}_{k}\left(S_{k-1}\right)=\text { busy } \mid \mathcal{O}_{k}(0)=\text { busy }\right)+ \\
& +P\left(\tau_{k} \leq \tau \mid\left(\mathcal{O}_{k}(0)=\text { busy } \cap \mathcal{O}_{k}\left(S_{k-1}\right)=i d l e\right) P\left(\mathcal{O}_{k}\left(S_{k-1}\right)=i d l e \mid \mathcal{O}_{k}(0)=\text { busy }\right)\right.
\end{aligned}
$$

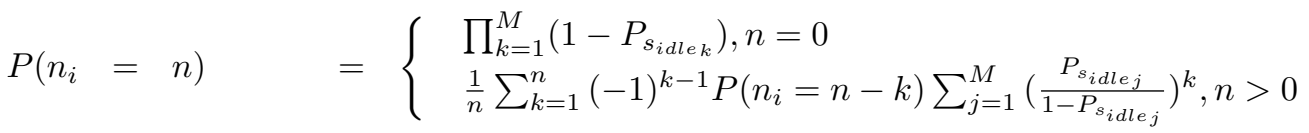

$$
\begin{aligned}
& P_{m d_{k}}=1-\sum_{i=1}^{l_{k}} P\left(l_{d}=i\right)=1-\sum_{i=1}^{l_{k}}\left(\frac{1}{i} \sum_{m=1}^{i}(-1)^{m-1} P\left(l_{d}=i-m\right) \sum_{j=1}^{l_{k}}\left(\frac{1-P_{m d_{k j}}}{P_{m d_{k j}}}\right)^{m}\right) \\
& P_{f a_{k}}=\sum_{i=1}^{l_{k}} P\left(l_{f a}=i\right)=\sum_{i=1}^{l_{k}}\left(\frac{1}{i} \sum_{m=1}^{i}(-1)^{m-1} P\left(l_{f a}=i-m\right) \sum_{j=1}^{l_{k}}\left(\frac{P_{f a_{k j}}}{1-P_{f a_{k j}}}\right)^{m}\right) \\
& P\left(T<T_{C P} \mid n_{i}=n\right)=P\left(\tau_{n} \leq T_{S}-S_{n-1}\right)=\int_{0}^{T_{S}} f_{\tau_{1}}\left(\tau_{1}\right) \cdots \int_{0}^{T_{S}-S_{n-2}} f_{\tau_{n-1}}\left(\tau_{n-1}\right) \int_{0}^{T_{S}-S_{n-1}} f_{\tau_{n}}\left(\tau_{n}\right) d \tau_{n} d \tau_{n-1} \cdots d \tau_{1}
\end{aligned}
$$

having $x$ successive periods, equals

$$
\mathbf{E}\left[T_{k}\right]=\sum_{x=0}^{\infty} P(X=x)\left((x-1) \mathbf{E}\left[T_{k}\right]_{C P}+\mathbf{E}\left[T_{k}\right]_{I N P}\right)
$$

Then, $\mathbf{E}\left[T_{k}\right]_{C P}$ can be expressed as

$$
\mathbf{E}\left[T_{k}\right]_{C P}=\sum_{n=1}^{M} P\left(n_{i}=n\right) \mathbf{E}\left[\tau_{k} \mid n_{i}=n\right]
$$

where $\mathbf{E}\left[\tau_{k} \mid n_{i}=n\right]$ is the expected operation time on the $k$ th visited channel, when having a complete period and there are $n$ licensed channels sensed idle, which can be expressed as in (30). The expression of $\mathbf{E}\left[T_{k}\right]_{I N P}$ is analogous to (29). However, as the distribution of $\tau_{k}$ differs for incomplete periods, for $\mathbf{E}\left[T_{k}\right]_{I N P}, \mathbf{E}\left[\tau_{k} \mid n_{i}=n\right]$ should be replaced by $\mathbf{E}\left[\tau_{k}^{\prime} \mid n_{i}=n\right]$, which is given by (31).

\section{ApPENDix C}

As proved in [22], the traffic-dependent parameters under saturated conditions are given by $P_{s_{k}}=N p(1-p)^{\bar{N}_{k}+N-1}$ and $P_{s_{k}}^{\prime}=\bar{N}_{k} p(1-p)^{N_{k}+N-1}$, where $N$ is the number of users of the SN, $\bar{N}_{k}$ the average number of SUs that belong to other SNs and operate on the $k$ th channel and $p$ the probability that a SU transmits in a randomly chosen slot time. The probability of an idle slot is given by $P_{i_{k}}=1-P_{t r_{k}}$, where $P_{t r_{k}}=1-(1-p)^{\bar{N}_{k}+N}$ denotes the probability that there is at least one transmission in the considered slot time. Then, we calculate the probabilities of collision by subtracting from the total collision probability derived in [22], the probability of a collision in which only the $\bar{N}_{k}$ SUs are involved for the calculation of $P_{c_{k}}$, and the probability of a collision in which only the $N$ SUs are involved for the calculation of $P_{c_{k}}^{\prime}$.

$$
P_{c_{k}}=P_{c_{k t o t}}-(1-p)^{N} \sum_{j=2}^{\bar{N}_{k}}\left(\begin{array}{c}
\bar{N}_{k} \\
j
\end{array}\right) p^{j}(1-p)^{\bar{N}_{k}-j}
$$

Note that all probabilities that concern the unlicensed channel operation are given by the equivalent ones for the licensed channels by substituting $\bar{N}_{k}=N_{\text {unlic }}$ and $N=1$. Finally, $\bar{Y}_{k}$ is 1 for centralized networks or different (e.g., equal to $\bar{X}_{k}$ ) for ad hoc networks, while $\bar{X}_{k}$ is given by (33).

\section{APPENDIX D \\ PROOF OF LEMMA 3}

As the process in the unlicensed channel will be repeated until at least one channel is sensed idle, the expected time spent in the unlicensed channel can be expressed as

$$
\begin{aligned}
\mathbf{E}\left[T_{U}\right] & =\sum_{i=0}^{\infty}\left(1-P_{U_{s}}\right)^{i} P_{U_{s}}\left(i \mathbf{E}\left[T_{U_{u n}}\right]+\mathbf{E}\left[T_{U_{s}}\right]\right) \\
& =\mathbf{E}\left[T_{U_{u n}}\right]\left(\frac{1}{P_{U_{s}}}-1\right)+\mathbf{E}\left[T_{U_{s}}\right]
\end{aligned}
$$

where $P_{U_{s}}=1-\prod_{n=1}^{M}\left(1-P_{s_{i_{\text {dle }}}}\right)$ is the probability that there is at least one licensed channel sensed idle, and $T_{U_{u n}}$, 


$$
\begin{array}{r}
\mathbf{E}\left[\tau_{k} \mid n_{i}=n\right]=\int_{0}^{T_{S}} f_{\tau_{1}}\left(\tau_{1}\right) \cdots \int_{0}^{T_{S}-S_{k-2}} f_{\tau_{k-1}}\left(\tau_{k-1}\right) \int_{T_{S}-S_{k-1}}^{\infty}\left(T_{S}-S_{k-1}\right) f_{\tau_{k}}\left(\tau_{k}\right) d \tau_{k} d \tau_{k-1} \cdots d \tau_{1} \\
+\cdots+\int_{0}^{T_{S}} f_{\tau_{1}}\left(\tau_{1}\right) \cdots \int_{0}^{T_{S}-S_{k-1}} \tau_{i} f_{\tau_{k}}\left(\tau_{k-1}\right) \cdots \int_{T_{S}-S_{n-1}}^{\infty} f_{\tau_{n}}\left(\tau_{n}\right) d \tau_{n} d \tau_{n-1} \cdots d \tau_{1} \\
\mathbf{E}\left[\tau_{k}^{\prime} \mid n_{i}=n\right]=\int_{0}^{T_{S}} f_{\tau_{1}}\left(\tau_{1}\right) \cdots \int_{0}^{T_{S}-S_{k-1}} \tau_{k} f_{\tau_{k}}\left(\tau_{k-1}\right) \cdots \int_{0}^{T_{S}-S_{n-1}} f_{\tau_{n}}\left(\tau_{n}\right) d \tau_{n} d \tau_{n-1} \cdots d \tau_{1} \\
\bar{X}_{k}=\frac{1}{P_{c_{k}}}\left[N p(1-p)^{N-1} \sum_{i=1}^{N_{k}}\left(\begin{array}{c}
N_{k} \\
i
\end{array}\right) p^{i}(1-p)^{N_{k}-i}+\sum_{j=2}^{N} j\left(\begin{array}{c}
N \\
j
\end{array}\right) p^{j}(1-p)^{N-j} \sum_{l=0}^{N_{k}}\left(\begin{array}{c}
N_{k} \\
l
\end{array}\right) p^{l}(1-p)^{N_{k}-l}\right]
\end{array}
$$

$T_{U_{s}}$ denote the time spent when there is no channel sensed idle and when there is at least one channel sensed idle, respectively. Regarding $\mathbf{E}\left[T_{U_{u n}}\right]$, it may be claimed, by inspecting Fig. 2, that $\mathbf{E}\left[T_{U_{u n}}\right]=\mathbf{E}\left[t_{p h 1}\right]+t_{p h 2}+t_{p h 3}$, where $\mathbf{E}\left[t_{p h 1}\right]$ is the expected duration of ph1 and $t_{p h 2}, t_{p h 3}$ the ph2, ph3 durations in the unlicensed channel. It is worth noting that, the difference between $\mathbf{E}\left[T_{U_{u n}}\right]$ and $\mathbf{E}\left[T_{U_{s}}\right]$ lies in the fact that the latter includes the additional time to switch to a licensed channel at the end of the process and thus $\mathbf{E}\left[T_{U_{s}}\right]=\mathbf{E}\left[T_{U_{u n}}\right]+t_{s w}$.

The expected duration of $t_{p h 1}$ can be split up into the contention time to gain access to the unlicensed channel, $T_{\text {cont }}$, and the constant time $T_{t r_{1}}$ that includes the time required to transmit the RFS and ACK packets, and the DIFS and SIFS waiting times. Therefore, $\mathbf{E}\left[t_{p h 1}\right]=\mathbf{E}\left[T_{\text {cont }}\right]+T_{t r_{1}}$, where $T_{t r_{1}}=t_{R F S}+t_{S I F S}+t_{A C K}+t_{D I F S}$. Then, the contention time can in turn be expressed as $\mathbf{E}\left[T_{\text {cont }}\right]=$ $\mathbf{E}\left[N_{\text {unsuc }}\right] \mathbf{E}\left[T_{\text {unsuc }}\right]$, where $\mathbf{E}\left[N_{\text {unsuc }}\right]$ denotes the expected number of unsuccessful slots until the cluster head gains access to the unlicensed channel and $\mathbf{E}\left[T_{\text {unsuc }}\right]$ the expected duration of an unsuccessful slot. Thus, $\mathbf{E}\left[N_{\text {unsuc }}\right]$ is equal to

$$
\mathbf{E}\left[N_{\text {unsuc }}\right]=\sum_{i=0}^{\infty} i\left(1-P_{s}\right)^{i} P_{s}=\frac{1}{P_{s}}-1
$$

where $P_{s}$ is the probability of having a successful transmission by the cluster head in the unlicensed channel. Then, $\mathbf{E}\left[T_{\text {unsuc }}\right]$ is given by

$$
\mathbf{E}\left[T_{\text {unsuc }}\right]=\frac{P_{i} \sigma+P_{s}^{\prime} T_{r s}+\left(P_{c}+P_{c}^{\prime}\right) T_{c}}{1-P_{s}}
$$

where $P_{i}$ is the probability of having an idle slot in the unlicensed channel; $P_{c}$ the probability of a collision slot of the SN under study in the unlicensed channel; $P_{s}^{\prime}$ and $P_{c}^{\prime}$ the probabilities of a successful transmission and collision slot, respectively, of the other SNs in the unlicensed channel; and $\sigma, T_{r s}$ and $T_{c}$ are the durations of an idle, a successful transmission and a collision slot. Finally, $t_{p h 2}=M_{u s} t_{s n}+\left(M_{u s}+1\right) t_{s w}$ and $t_{p h 3}=t_{B}+t_{S R}(N-1)+t_{L I S T}+t_{S I F S} N+t_{D I F S}$, where $M_{u s}$ is the number of licensed channels sensed by each $\mathrm{SU}, t_{s n}$ the time to sense a channel, and $t_{B}, t_{S R}, t_{L I S T}$ the time required for beacon, report and list packets transmission, respectively.

\section{APPENDIX E}

Similarly to the calculation of the time devoted to data transmissions, the time devoted to detect the resumption of the PUs' activity and switch to another channel may be written as

$$
\mathbf{E}\left[T_{r s n}\right]=\sum_{x=0}^{\infty} P(X=x)\left((x-1) \mathbf{E}\left[T_{r s n}\right]_{C P}+\mathbf{E}\left[T_{r s n}\right]_{I N P}\right)
$$

where $\mathbf{E}\left[T_{r s n}\right]_{C P}, \mathbf{E}\left[T_{r s n}\right]_{I N P}$ denote the expected time spent in the reaction periods and the sensing procedures in a complete and an incomplete period, respectively. Then, $\mathbf{E}\left[T_{r s n}\right]_{C P}$ can be expressed as

$$
\mathbf{E}\left[T_{r s n}\right]_{C P}=\sum_{k \in \mathcal{B}} P_{k} \mathbf{E}\left[T_{s n_{k}}\right]+\sum_{k \in \mathcal{B}} P_{k}(k-1) \delta+t_{s w}
$$

where $P_{k}$ is the probability of having a sensing period in the $k$ th channel, namely the probability of having a complete period in the $k$ th channel, $M$ is the number of sensed channels, $n_{i}$ is the number of channels sensed idle, and $\mathbf{E}\left[T_{s n_{k}}\right]$ the expected duration of the sensing procedure in the $k$ th licensed channel. $P_{k}$ is given by (39) and $\mathbf{E}\left[T_{s n_{k}}\right]=\mathbf{E}\left[t_{p h 1_{k}}\right]+t_{p h 2}+$ $t_{p h 3}$, where $\mathbf{E}\left[t_{p h 1_{k}}\right]$ is calculated as $\mathbf{E}\left[t_{p h 1}\right]$ but taking into account the respective probabilities of the $k$ th channel. Finally, $\mathbf{E}\left[T_{r s n}\right]_{I N P}=\sum_{n=1}^{M} P\left(n_{i}=n\right) n \delta$.

\section{APPENDIX F PROOF OF LEMMA 4}

Following the rationale of (34), $\mathbf{E}\left[E_{U}\right]$ can be expressed as

$$
\mathbf{E}\left[E_{U}\right]=E_{U_{u n}}\left(\frac{1}{P_{U_{s}}}-1\right)+E_{U_{s}}
$$

where $E_{U_{s}}=\mathbf{E}\left[E_{p h 1}\right]+E_{p h 2}+E_{p h 3}+N \boldsymbol{P}_{s w} t_{s w}$ is the energy consumption in the unlicensed channel when there is at least one licensed channel sensed idle and $E_{U_{u n}}=$ $\mathbf{E}\left[E_{p h 1}\right]+E_{p h 2}+E_{p h 3}$ otherwise. $\boldsymbol{P}_{s w}$ is the power to switch channel and $\mathbf{E}\left[E_{p h 1}\right], E_{p h 2}, E_{p h 3}$ the expected energy consumed in $t_{p h 1}, t_{p h 2}$ and $t_{p h 3}$, respectively. Then, $\mathbf{E}\left[E_{p h 1}\right]=\mathbf{E}\left[N_{\text {unsuc }}\right] \mathbf{E}\left[E_{\text {unsuc }}\right]+E_{t r_{1}}$, where $\mathbf{E}\left[N_{\text {unsuc }}\right]$ calculated in (35) and $\mathbf{E}\left[E_{\text {unsuc }}\right]$ is the expected energy consumed in an unsuccessful slot, while $E_{t r_{1}}$ the one during $T_{t r_{1}}$. E $\left[E_{\text {unsuc }}\right]$ is given by (41), where $\boldsymbol{P}_{t r s m}, \boldsymbol{P}_{r e c}$ and $\boldsymbol{P}_{i d l e}$ are the transmission, reception and idle powers. Then, 


$$
\begin{aligned}
P_{k} & =\int_{0}^{T_{S}} f_{\tau_{1}}\left(\tau_{1}\right) \cdots \int_{0}^{T_{S}-S_{k-2}} f_{\tau_{k-1}}\left(\tau_{k-1}\right) \int_{T_{S}-S_{k-1}}^{\infty} f_{\tau_{k}}\left(\tau_{k}\right) d \tau_{k} d \tau_{k-1} \cdots d \tau_{1} \\
\mathbf{E}\left[E_{\text {unsuc }}\right] & =\frac{N \boldsymbol{P}_{\text {idle }}\left(P_{i} \sigma+P_{s}^{\prime} T_{r s}+P_{c}^{\prime} T_{c}\right)+P_{c}\left(t_{d a t a}\left(\boldsymbol{P}_{\text {trsm }}+(N-1) \boldsymbol{P}_{r e c}\right)+N \boldsymbol{P}_{\text {idle }} t_{D I F S}\right)}{1-P_{s}}
\end{aligned}
$$

$E_{t r_{1}}=t_{R F S}\left(\boldsymbol{P}_{t r s m}+(N-1) \boldsymbol{P}_{r e c}\right)+t_{A C K}\left(\boldsymbol{P}_{t r s m}+\boldsymbol{P}_{r e c}+\right.$ $\left.(N-2) \boldsymbol{P}_{\text {idle }}\right)+N \boldsymbol{P}_{\text {idle }}\left(t_{D I F S}+t_{S I F S}\right)$. Finally, the energy consumptions during the time periods $t_{p h 2}$ and $t_{p h 3}$ are equal to $E_{p h 2}=N\left(M_{u s} \boldsymbol{P}_{s n} t_{s n}+\left(M_{u s}+1\right) \boldsymbol{P}_{s w} t_{s w}\right)$, with $\boldsymbol{P}_{s n}$ denoting the sensing power, and $E_{p h 3}=\left(\boldsymbol{P}_{\text {trsm }}+(N-\right.$ 1) $\left.\boldsymbol{P}_{r e c}\right)\left(t_{B}+t_{L I S T}\right)+t_{S R}(N-1)\left(\boldsymbol{P}_{\text {trsm }}+\boldsymbol{P}_{r e c}+(N-\right.$ 2) $\left.\boldsymbol{P}_{i d l e}\right)+N \boldsymbol{P}_{i d l e}\left(t_{D I F S}+t_{S I F S} N\right)$.

\section{APPENDIX G}

The expected energy consumption in the reaction periods and sensing procedures equals to

$\mathbf{E}\left[E_{r s n}\right]=\sum_{x=0}^{\infty} P(X=x)\left((x-1) \mathbf{E}\left[E_{r s n}\right]_{C P}+\mathbf{E}\left[E_{r s n}\right]_{I N P}\right)$

where $\mathbf{E}\left[E_{r s n}\right]_{C P}, \mathbf{E}\left[E_{r s n}\right]_{I N P}$ are the expected energy consumptions both in the reaction periods and the sensing procedures in a complete and an incomplete period, respectively, and equal to

$$
\begin{gathered}
\mathbf{E}\left[E_{r s n}\right]_{C P}=\sum_{k \in \mathcal{B}} P_{k} \mathbf{E}\left[E_{s n_{k}}\right]+\sum_{k \in \mathcal{B}} P_{k}(k-1) E_{\delta}+E_{s w} \\
\mathbf{E}\left[E_{r s n}\right]_{I N P}=\sum_{n=1}^{M} P\left(n_{i}=n\right) n E_{\delta}
\end{gathered}
$$

where $E_{\delta}=N\left(\boldsymbol{P}_{r e c} t_{r}+\boldsymbol{P}_{s w} t_{s w}\right)$ and $\mathbf{E}\left[E_{s n_{k}}\right]=\mathbf{E}\left[E_{p h 1_{k}}\right]+$ $E_{p h 2}+E_{p h 3_{k}}$ are the energy consumptions during $\delta$ and the sensing procedures in the licensed channels, respectively. $\mathbf{E}\left[E_{p h 1_{k}}\right]$ is calculated as $\mathbf{E}\left[E_{p h 1}\right]$ but taking into account the respective probabilities of the $k$ th channel. Then, $\mathbf{E}\left[E_{\text {cont }_{k}}\right]$ may be expressed as $\mathbf{E}\left[E_{\text {cont }_{k}}\right]=\mathbf{E}\left[E_{s_{k}}\right]+\mathbf{E}\left[E_{c_{k}}\right]+\mathbf{E}\left[E_{i_{k}}\right]$, where $\mathbf{E}\left[E_{s_{k}}\right]=\beta P_{s_{k}} E_{s}, \mathbf{E}\left[E_{c_{k}}\right]=\beta P_{c_{k}} E_{c}$ and $\mathbf{E}\left[E_{i_{k}}\right]=$ $\beta N \boldsymbol{P}_{\text {idle }}\left(P_{i_{k}} \sigma+P_{s_{k}}^{\prime} T_{r s}+P_{c_{k}}^{\prime} T_{c}\right)$ are the expected energies consumed in the successful transmissions, collisions or idle slots of the $\mathrm{SN}$ on the $k$ th channel, with $\beta=\mathbf{E}\left[T_{k}\right] / \mathbf{E}\left[T_{\text {slot }}\right]$ and $E_{s}, E_{c}$ the energy consumed in a successful transmission, collision slot of the $\mathrm{SN}$, respectively, and are given by $E_{s}=\left(t_{d a t a}+t_{A C K}\right)\left(\boldsymbol{P}_{\text {trsm }}+\boldsymbol{P}_{\text {rec }}+(N-2) \boldsymbol{P}_{i d l e}\right)+$ $N \boldsymbol{P}_{i d l e}\left(t_{D I F S}+t_{S I F S}\right)$ and $E_{c}=t_{d a t a}\left(\bar{X}_{k} \boldsymbol{P}_{\text {trsm }}+\bar{Y}_{k} \boldsymbol{P}_{r e c}+\right.$ $\left.\left(N-\bar{X}_{k}-\bar{Y}_{k}\right) \boldsymbol{P}_{i d l e}\right)+N \boldsymbol{P}_{i d l e} t_{D I F S}$, where $\bar{X}_{k}, \bar{Y}_{k}$ are the average numbers of SUs of the SN that are involved in a collision in transmission and reception mode, respectively.

\section{ACKNOWLEDGMENT}

This work has been funded by the Research Projects GREENET (PITN-GA-2010-264759), GEOCOM (TEC201127723-C02-01), and CROSSFIRE (MITN-317126).

\section{REFERENCES}

[1] J. Mitola et al., "Cognitive radio: making software radios more personal," IEEE Pers. Commun., vol. 6, no. 4, pp. 13-18, Aug. 1999.

[2] S. Haykin, "Cognitive radio: brain-empowered wireless communications," IEEE J. Sel. Areas Commun., vol. 23, no. 2, pp. 201-220, Feb. 2005.

[3] I. F. Akyildiz et al., "NeXt generation / dynamic spectrum access / cognitive radio wireless networks: a survey," Comput. Netw. J. (Elsevier), vol. 50, pp. 2127-2159, Sep. 2006.

[4] B. Wang and K. J. Ray. Liu, "Advances in cognitive radio networks: a survey,” IEEE J. Sel. Topics Signal Process., vol. 5, no. 1, pp. 5-23, Feb. 2011.

[5] F. Adelantado et al., "Adaptive sensing user selection mechanism in cognitive wireless networks," IEEE Commun. Lett., vol. 14, no. 9, pp. 800-802, Sep. 2010.

[6] I. F. Akyildiz et al., "Cooperative spectrum sensing in cognitive radio networks: a survey," Phys. Commun., vol. 4, no. 1, pp. 40-62, Mar. 2011.

[7] E. C. Y. Peh et al., "Energy-efficient cooperative spectrum sensing in cognitive radio networks," in Proc. IEEE Global Telecommun. Conf. (GLOBECOM), Dec. 2011.

[8] R. Misra and A. P. Kannu, "Optimal sensing-order in cognitive radio networks with cooperative centralized sensing," in Proc. IEEE Int. Conf. Commun. (ICC), Jun. 2012.

[9] H. Jiang et al., "Optimal selection of channel sensing order in cognitive radio," IEEE Trans. Wireless Commun., vol. 8, no. 1, pp. 297-307, Jan. 2009.

[10] Z. Khan et al., "Autonomous sensing order selection strategies exploiting channel access information," IEEE Trans. Mobile Comput., vol. 12, no. 2, pp. 274-288, Feb. 2013

[11] B. Gao et al., "A taxonomy of coexistence mechanisms for heterogeneous cognitive radio networks operating in TV white spaces," IEEE Wireless Commun., vol. 19, no. 4, pp. 41-48, Aug. 2012.

[12] A. Mesodiakaki et al., "Energy-efficient contention-aware channel selection in cognitive radio ad-hoc networks," in Proc. IEEE Int. Workshop Comput. Aided Modeling Design Commun. Links Netw. (CAMAD), Sep. 2012.

[13] C. Sun et al., "Optimizing the coexistence performance of secondaryuser networks under primary-user constraints for dynamic spectrum access," IEEE Trans. Veh. Technol., vol. 61, no. 8, pp. 3665-3676, Oct. 2012.

[14] K. Ishizu et al., "TV white space database for coexistence of primarysecondary and secondary-secondary systems in mesh networking," in Proc. Int. Symp. Wireless Pers. Multimedia Commun. (WPMC), Sep. 2012.

[15] D. T. C. Wong and F. Chin, "Sensing-saturated throughput performance in multiple cognitive CSMA/CA networks," in Proc. IEEE Veh. Technol. Conf. (VTC), May 2010.

[16] Y. Zhao et al., "FMAC: a fair MAC protocol for coexisting cognitive radio networks," in Proc. IEEE INFOCOM, Apr. 2013.

[17] L. Le and E. Hossain, "OSA-MAC: a MAC protocol for opportunistic spectrum access in cognitive radio networks," in Proc. IEEE Wireless Commun. Netw. Conf. (WCNC), Mar./Apr. 2008.

[18] M. Hossian et al., "Channel ranking algorithms for cognitive coexistence of IEEE 802.15.4," in Proc. IEEE Int. Symp. Pers. Indoor Mobile Radio Commun. (PIMRC), Sep. 2009.

[19] N. Jain and S. Das, "A multichannel CSMA MAC protocol with receiverbased channel selection for multihop wireless networks," in Proc. Int. Conf. Comput. Commun. Netw. (ICCCN), Oct. 2001.

[20] D. Nam and H. Min "An energy-efficient clustering using a round-robin method in a wireless sensor network," Proc. ACIS Int. Conf. Softw. Eng. Res., Manag. \& Applicat. (SERA), Aug. 2007. 
[21] H. Harada and I. Tinnirello, "A small-size software defined cognitive radio prototype," in Proc. IEEE Int. Symp. Pers. Indoor Mobile Radio Commun. (PIMRC), Sep. 2008.

[22] G. Bianchi, "Performance analysis of the IEEE 802.11 distributed coordination function," IEEE J. Sel. Areas Commun., vol. 18, no. 3, pp. 535-547, Mar. 2000.

[23] L. Thanh Tan and L. Bao Le "Channel assignment with access contention resolution for cognitive radio networks," IEEE Trans. Veh. Technol., vol. 61, no. 6, pp. 2808-2823, Jul. 2012.

[24] W. Wang et al., "Channel assignment schemes for cooperative spectrum sensing in multi-channel cognitive radio networks," Wireless Commun. Mobile Comput., Oct. 2013. doi: 10.1002/wcm.2442

[25] J. Kuri and S. K. Kasera, "Reliable multicast in multi-access wireless LANs," in Proc. IEEE INFOCOM, Mar. 1999.

[26] J. Lunden et al., "Collaborative cyclostationary spectrum sensing for cognitive radio systems," IEEE Trans. Signal Process., vol. 57, no. 11, pp. 4182-4195, Nov. 2009.

[27] "Part 11: Wireless LAN medium access control and physical layer specifications,” IEEE Standard 802.11, 2012.

[28] S. Rayanchu et al., "Diagnosing wireless packet losses in 802.11: separating collision from weak signal," in Proc. IEEE INFOCOM, Apr. 2008.

[29] L. Zhao et al., "Using incompletely cooperative game theory in wireless mesh networks," IEEE Network, vol. 22, no. 1, pp. 39-44, Jan./Feb. 2008.

[30] D. Malone et al., "Modeling the 802.11 distributed coordination function in non-saturated heterogeneous conditions," IEEE/ACM Trans. Netw., vol. 15, no. 1, pp. 159-172, Feb. 2007.

[31] H. Kim and K. G. Shin, "In-band spectrum sensing in IEEE 802.22 WRANs for incumbent protection," IEEE Trans. Mobile Comp., vol. 9, no. 12 , pp. $1766-1779$, Dec. 2010

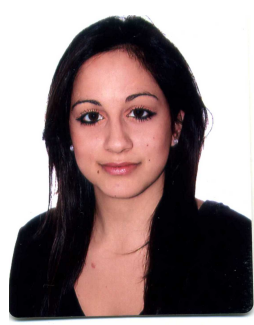

Agapi Mesodiakaki was born in Heraklion (Crete, Greece) in 1987. She obtained her five-year diploma (MSc) in Electrical and Computer Engineering in National Technical University of Athens (NTUA) in February 2011. During her studies, she accomplished a couple of internships in Hellenic Telecommunications Organization (OTE S.A) in Greece and in TU Darmstadt in Germany. Currently, she is a Marie-Curie Early-Stage-Researcher, pursuing a Ph.D. degree at the Signal Theory and Communications Department (TSC) of the Universitat Politcnica de Catalunya (UPC) in Barcelona. Her main research interests include energyefficient radio resource management (RRM) techniques for wireless communication networks, green cognitive radio networks and security algorithms.

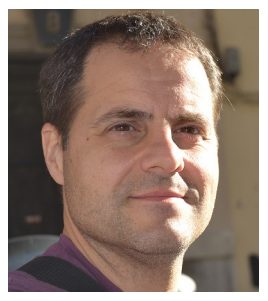

Luis Alonso obtained the Ph.D. from UPC (Barcelona) in 2001 and got a permanent tenured position at the same University becoming an Associate Professor in 2006. He has been co-founder of the Wireless Communications and Technologies Research Group (WiComTec), to which currently belongs. His current research interests are within the field of medium access protocols, radio resource management, cross-layer optimization, cooperative transmissions, cognitive radio and QoS features for all kind of wireless communications systems. He is author of forty research papers in international journals and magazines, one book, twelve chapters of books and more than one hundred papers in international congresses and symposiums. He has received several best paper awards.

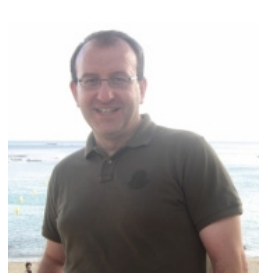

Christos Verikoukis got his Ph.D. from the Technical University of Catalonia in 2000. He is currently a Senior Researcher and the Head of the SMARTECH department at CTTC and an adjunct professor at UB. His area of expertise is in the design of energy efficient layer 2 protocols and RRM algorithms for short-range wireless cooperative and network coded communications. He has published 63 journal papers and over 130 conference papers. He is also coauthor in 2 books, 14 chapters in different books and in 2 patents. He has supervised 15 Ph.D. students and 5 Post Docs researchers since 2004. Dr. Verikoukis has participated in more than 30 competitive projects while he has served as the Principal investigator in national projects in Greece and Spain as well as the technical manager in Marie-Curie and Celtic projects. He has appointed to serve as a reviewer in FP7 projects funded by the European Commission and as an EU-independent expert acting evaluator in different FP7 funding mechanisms. He was General Chair of the 17th and 18th IEEE Workshop on ComputerAided Modeling, Analysis and Design of Communication Links and Networks (CAMAD), and the TPC Co-Chair of the 15th IEEE International Conference on E-health Networking, Application \& Services (Healthcom) and the 6th IEEE Latin-American Conference on Communications. He is currently officer (Secretary) of the IEEE ComSoc Technical Committee on Communication Systems Integration and Modeling (CSIM). Dr. Verikoukis received the best paper award of the Communication QoS, Reliability \& Modeling Symposium (CQRM) symposium in the IEEE ICC11 conference and the EURASIP 2013 Best Paper Award for the Journal on Advances in Signal Processing.

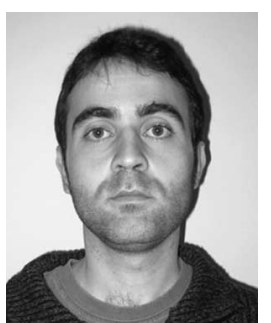

Ferran Adelantado was born in Barcelona in 1978 $\mathrm{He}$ received the degree in Telecommunications Engineering from Universitat Politcnica de Catalunya (UPC) in December 2001. In June 2002 he joined the Radio Communications Research Group in UPC, where he completed his Ph.D. in May 2007. Since December 2007 he has been working as an Associate professor at Universitat Oberta de Catalunya (UOC). Also from Universitat Oberta de Catalunya (UOC), he received the BS. in Business Sciences in July 2012. He has participated in several funded European and National projects, such as SOFIA, COSMOS, EVEREST, CAUTION, AlgotithmsForRealRouting or CROSSFIRE. His research interests include wireless networks, particularly MAC protocols and RRM techniques. Currently, his research is focused on LTE-A, cognitive and/or cooperative wireless networks, and self-organizing wireless networks. 